\title{
Dynamics of flow in a branching channel
}

\author{
Václav Uruba ${ }^{1,2,{ }^{*}}$, Pavel Procházka ${ }^{1}$, and Vladislav Skála ${ }^{1}$ \\ ${ }^{1}$ Institute of Thermomechanics of the Czech Academy of Sciences, Dolejškova 5, Praha 8, Czech Republic \\ ${ }^{2}$ UWB, Faculty of Mech. Eng., Depart. of Power System Engineering, Universitní 8, Plzen, Czech Republic
}

Received: 29 March 2019 / Accepted: 12 February 2021

\begin{abstract}
Flow in a branched channel is studied experimentally using the PIV technique. The presented study is concentrated on clarifying the dynamical behaviour in individual branches. The 11 branches issuing from the main channel perpendicularly, all channels are of rectangular cross-section. First, the time-mean flow-field is shown, then the flow dynamics is investigated using the OPD method. Flow patterns and frequencies are evaluated in three selected branches. The separated flow in branches exhibits highly dynamical behaviour, which differs substantially in the branches close to the inflow, in the main channel middle and close to its end. The typical topologies and frequencies of the detected quasi-periodical structures in the channel braches are shown in the study. Mostly, the flow-fields are populated by trains of vortices with alternating orientation and saddle-like structures. The flow-field close to the channel walls affects heat transfer process between the wall and fluid.
\end{abstract}

Keywords: Branching channel / turbulence / dynamics / OPD

\section{Introduction}

The branched channels are applied in many practical situations. In general, the main goal of branching is distribution of the flowing fluid across the various locations. However, the flow-rate distribution across the branches is irregular even if theirs geometry is similar - see e.g. [1].

There are extensive knowledge resources in available literature, many authors address this problem both from theoretical and practical point of view. In the classical engineering book [2] there are a lot of variants and configurations. Many papers deal with the flow-pattern in channels of rectangular cross-section. The so called "secondary flow of second kind" in a channel of square or rectangular cross-section was first observed by Nikuradse [3] and has been studied both experimentally [4] and theoretically [5]. Recently there are many numerical studies on the problem with detailed analysis of the flow-field available, see e.g. [6,7]. However, the flow-field dynamics is not studied in this case, the authors of the presented paper recently published the paper [8], where some preliminary results are shown. The novelty of the present study is detailed analysis of dynamical structures in the channel branches. The typical topologies and frequencies are to be presented.

The presented study was motivated by geometry of cooling channels in rotor of a power generator.

\footnotetext{
* e-mail: uruba@it.cas.cz
}

\section{Experimental setup}

The experimental model was designed and fabricated from the Plexiglas to allow optical access to the flow. Experiments were performed using the time-resolved PIV technique.

\subsection{Channel geometry}

The main channel was of the cross-section $25 \times 30 \mathrm{~mm}^{2}$, $1450 \mathrm{~mm}$ long with the dead-end. The 13 branches (A, B, ..., M) of reduced cross-sections $38 \times 4 \mathrm{~mm}^{2}$ and $127 \mathrm{~mm}$ in length, are distributed regularly along the main channel perpendicularly to the main channel axis, as shown in schematic picture in Figure 1.

The 3 channels were selected for detailed analysis, the first branch A, G in the main channel middle and the last but one $\mathrm{L}$. The selected branches are denoted by red letters in Figure 1.

In the inlet, fully turbulent and developed channel flow was present. The air flow in the main channel inlet is characterized by Reynolds number of 11300 , velocity of the air in the input is about $6.4 \mathrm{~m} / \mathrm{s}$. The volumetric flow is distributed into individual branches, the flow-rates $V$ are shown in Figure 2.

Detailed description of the channel modelused for the experiments is given in $[1,9]$.

Schematics of a singlebranch under study is in Figure 3, Cartesian coordinate system is introduced for each branch. The measuring plane was located in the mid-span of each 


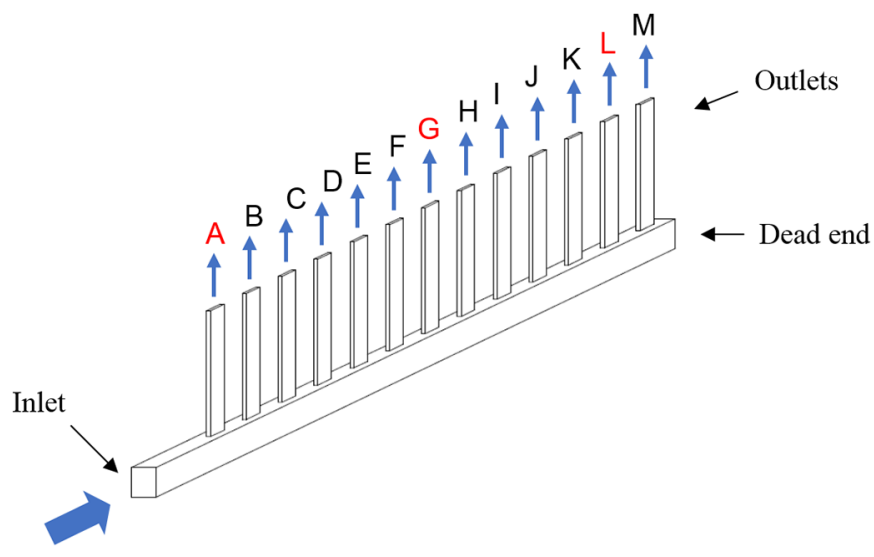

Fig. 1. Schematics of the channel.

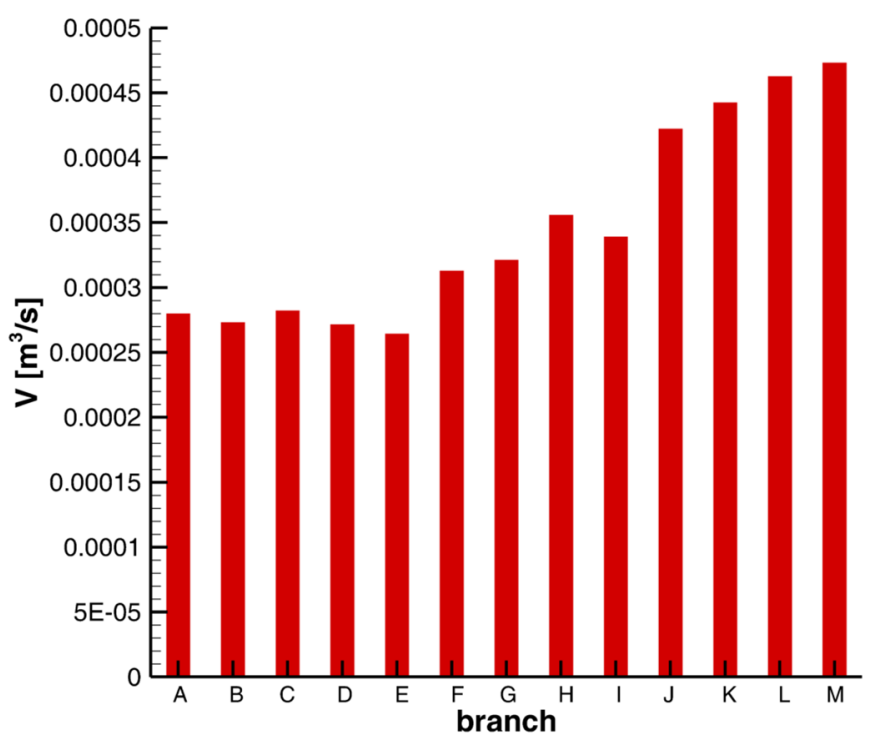

Fig. 2. Branches flow-rates.

channel. The area of a branch was divided into 2 parts: zones I and II for technical reasons, the zones are interconnected in the $x=65 \mathrm{~mm}$.

The coordinates in all graphs presented in this paper are given in millimetres.

\subsection{Instrumentation}

The time-resolved PIV method was used for the experiments in the channel model. The measuring system DANTEC consists of the double-pulse laser with cylindrical optics and CMOS camera. The software Dynamic Studio 3.4 was used for velocity-fields evaluation. Laser New Wave Pegasus Nd:YLF, double head, wavelength $527 \mathrm{~nm}$, maximal frequency $10 \mathrm{kHz}$, a shot energy is $10 \mathrm{~mJ}$ for $1 \mathrm{kHz}$ (corresponding power $10 \mathrm{~W}$ per head). Camera Phantom V711 with maximal resolution $1280 \times 800$ pixels and corresponding maximal frequency 3000 double-snaps per second. For the presented measurements, the frequency $1 \mathrm{kHz}$ and 4000 double-snaps in sequence corresponding to $4 \mathrm{~s}$ of record for mean evaluation was acquired. As particles

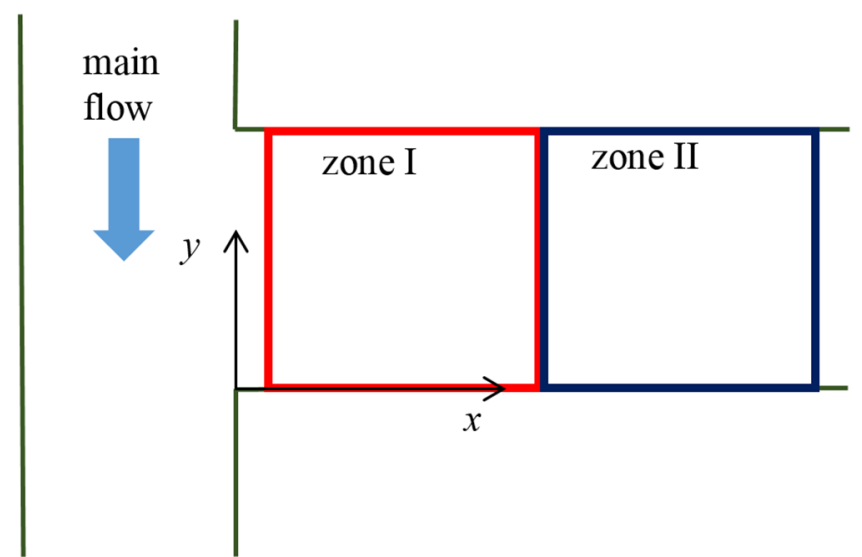

Fig. 3. Schematics of a branch, zones I and II are defined.

the oil droplets generated by SAFEX fog generator were used.

Detailed description of the measuring system could be find e.g. in [1].

\subsection{Analysis methods}

Instantaneous velocity fields in the measuring plane have been evaluated using "Adaptive PIV" method. The averaged velocity field has been evaluated first to obtain the flow statistics and remove the mean flow. Mean flow structure is evaluated as well as velocity components variances and correlation coefficient distributions.

For study of dynamical properties of the flow-field the Oscillation Pattern Decomposition method (OPD) was adopted resulting in series of OPD mode. Each OPD mode is characterized by topology in complex form (consisting of real and imaginary parts), frequency and attenuation of the pseudo-periodic (oscillating) behaviour. Attenuation or amplitude decay is described by e-folding time representing mean time period of the mode amplitude decay by "e". The other decay characteristic is "periodicity" which express the e-folding time in multiples of periods.

The details on OPD method could be find in [9].

\section{Results}

The results are divided into two parts. The first part is oriented on time-mean results (chapter 3.1), while the second part characterizes the flow dynamics in the 3 selected branches (chapter 3.2).

The zones I and II are presented separately as the dynamical analysis using the OPD method was applied separately to both zones. However the time-mean characteristics presented in the next chapter 3.1 follow-up between the zones satisfactorily.

\subsection{Time-mean characteristics}

The averaged characteristics will be presented in each figure both for the zone I on the left-hand the zone II on the 
Al

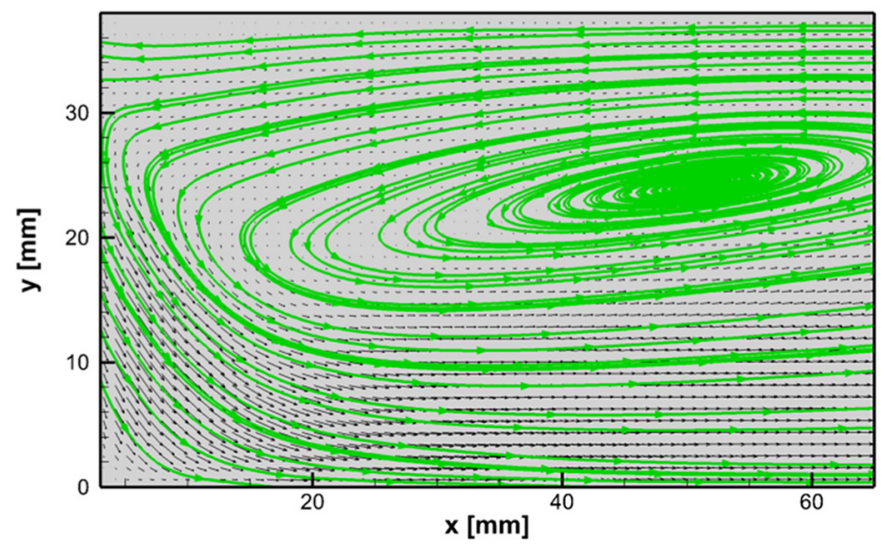

(a)

Gl

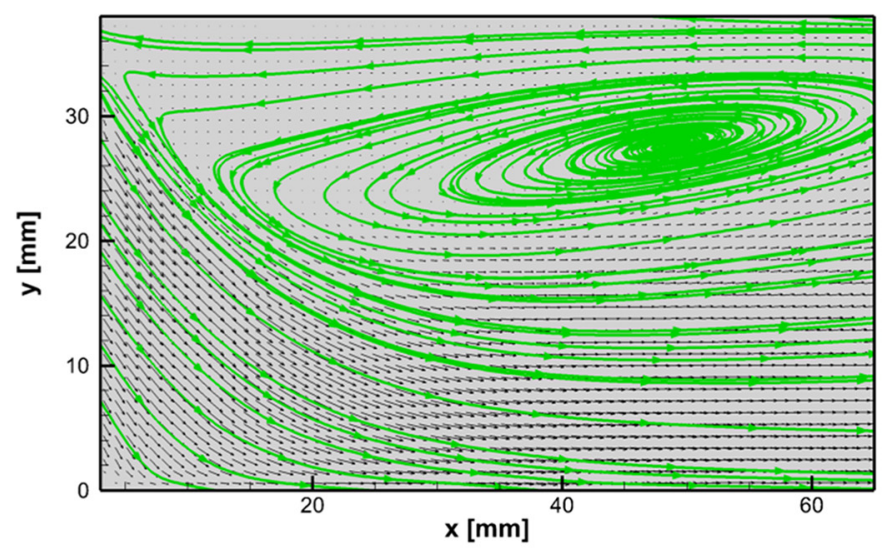

(c)

LI

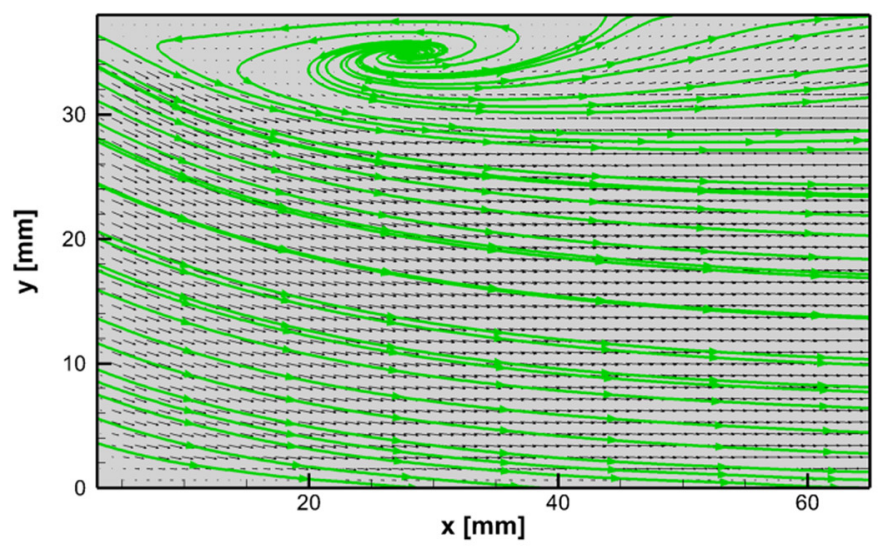

(e)
All

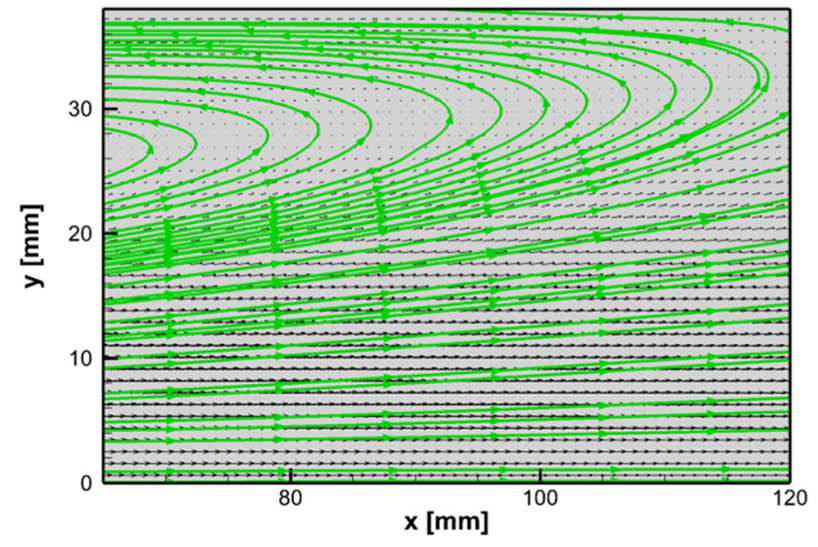

(b)

G॥

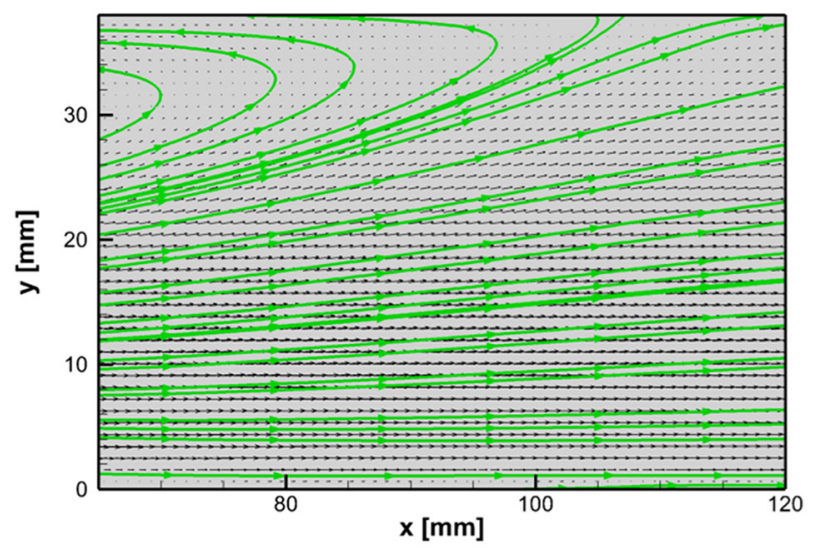

(d)

LII

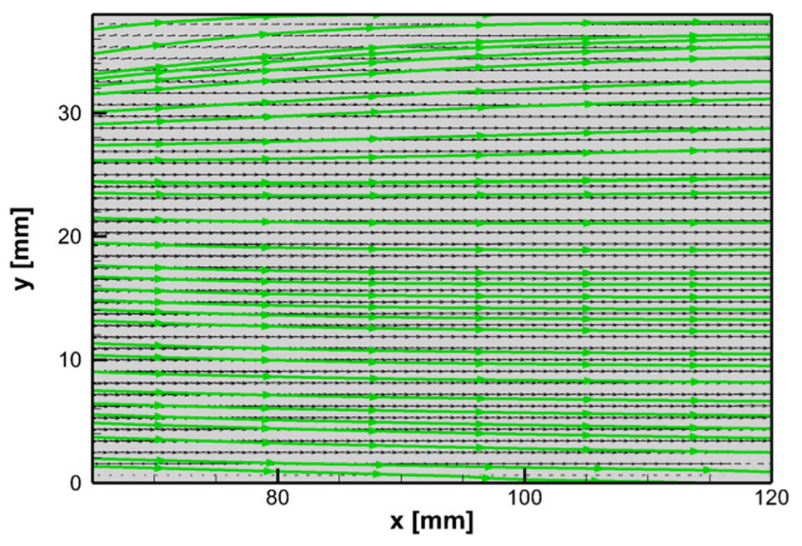

(f)

Fig. 4. Mean velocity vector fields in the branches A, G and L respectively: (a) AI, (b) AII, (c) GI, (d) GII, (e) LI and (f) LII.

right-hand sides, the division value of the $x$ coordinate is $65 \mathrm{~mm}$. The distributions of mean characteristics follow from one zone to the other satisfactorily. Please note that the dimensions of a branch are $127 \times 38 \mathrm{~mm}^{2}$.
The presentation of results starts with the mean velocities distributions, for the sake of clarity the vectorlines in green are added arbitrarily.

The mean velocity vector fields are shown in Figure $4 \mathrm{a}-\mathrm{f}$. 


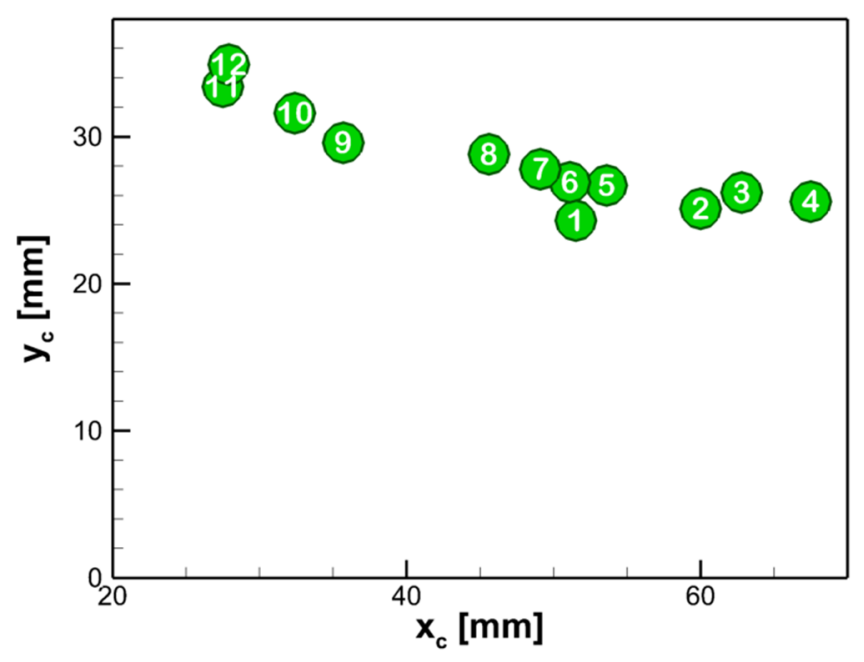

Fig. 5. Positions of mean flow vortex in individual branches A-M marked 1-13.

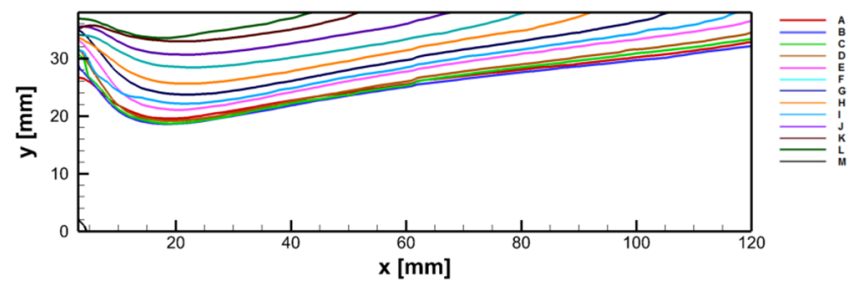

Fig. 6. Limits of the back-flow regions.

In the mean-flow fields the back-flow regions associated with a vortex located in left-hand part I could be detected. The back-flow results from separation of the flow on the sharp corner in $[0,38]$ position on the branch inlet from the main channel. This phenomenon was studied in details for all branches. The positions of the vortices centres $\left[x_{\mathrm{c}} ; y_{\mathrm{c}}\right]$ have been evaluated for all branches. The results are shown in Figure 5, the branches are marked with numbers 1-13. The branch $\mathrm{A}$ is marked with $1, \mathrm{G}$ with 7 and $\mathrm{L}$ with 12 respectively. In the last branch $\mathrm{M}$ (no. 13) no back-flow and associated vortex were detected.

The back-flow regions were identified within the branches. In Figure 6 the lines dividing the flow-field into back- and forward-flow regions are shown. The back-flow is locatedin the area above the limit line, below it there is forward flow. The limit line is defined as zero $x$-component mean velocity location.

The positions of the reattachment points on the upper branch wall are detected for branches $\mathrm{E}-\mathrm{L}$. In the branches A-D the flow does not reattach the upper wall, while in the last branch $\mathrm{M}$ no separation and thus no reattachment occur. In Figure 7 the $x_{\text {ra }}$ positions are shown.

To indicate regions with high dynamical behaviour the sum of variances $x$ and $y$ velocity components is to be presented next in Figure 8a-f. In Figures the dark-blue colour represents low dynamical activity regions, the red colour corresponds to excessive dynamics due to frequent errors in velocity evaluation using the standard PIV

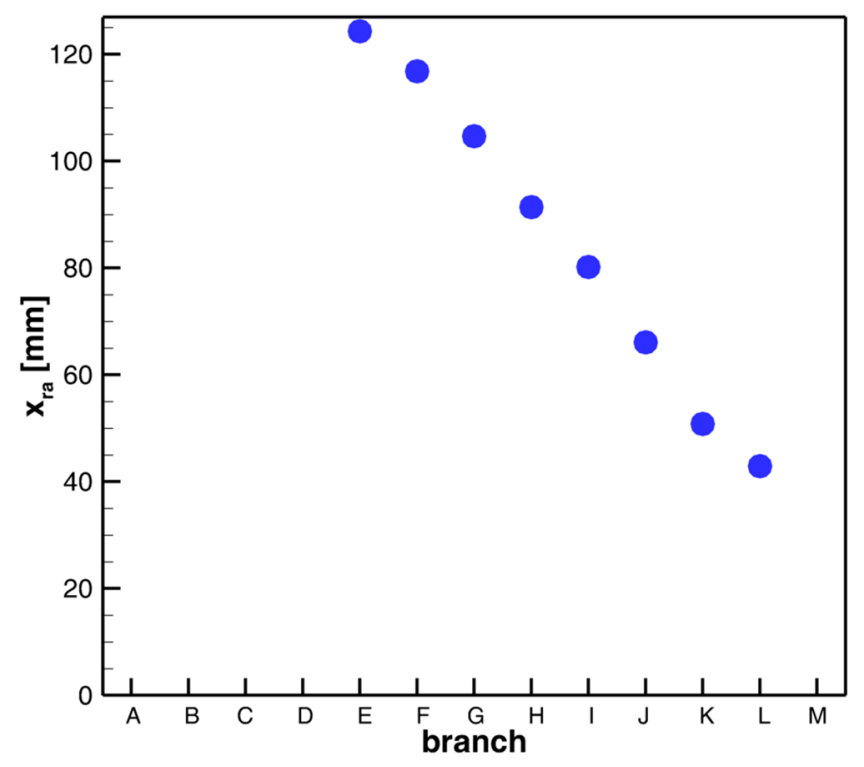

Fig. 7. Positions of the reattachment point.

evaluation procedure in near-wall regions. Finally, the light-blue and green regions are those with high dynamical activity in the flow itself.

To estimate turbulence generation, the correlation coefficient of the velocity components is evaluated in Figure $9 \mathrm{a}-\mathrm{f}$.

The correlation coefficient value close to 0 indicates low or even no turbulence generation, while negative (or positive) values approaching \pm 1 indicate turbulence production regions. The sign of the correlation depends on the coordinate system definition.

\subsection{Dynamics}

The OPD method has been applied on the zones I and II of the selected branches respectively. The analysis of both zones is completely independent to each other and OPD modes from one zone could not be linked directly to those of the other zone.

In Figure 10 there are OPD modes frequency and periodicity combinations evaluated by the standard procedure for the branch $\mathrm{A}$. The results for the zone I are in blue, while the red points indicate the zone II. Numbers of the modes ordered according to the e-folding time (descending order) are indicated within the points.

The periodicity values for the zone I are very low, this indicates that the modes decay very quickly in time. The value of periodicity 0.3 is considered to be the marginal value, below it the process could be considered as aperiodic, as the amplitude decay in one period is more than 10 - see [9]. On the other hand in the zone II the selected modes show much higher periodicity, higher than 2 , the modes are well periodical and relatively very stable.

A few modes have been selected with the highest value of periodicity for the detailed analysis. Those modes could be considered as oscillating or pseudo-periodic, the others, with low periodicity are decaying as they decay too quickly. 
Al

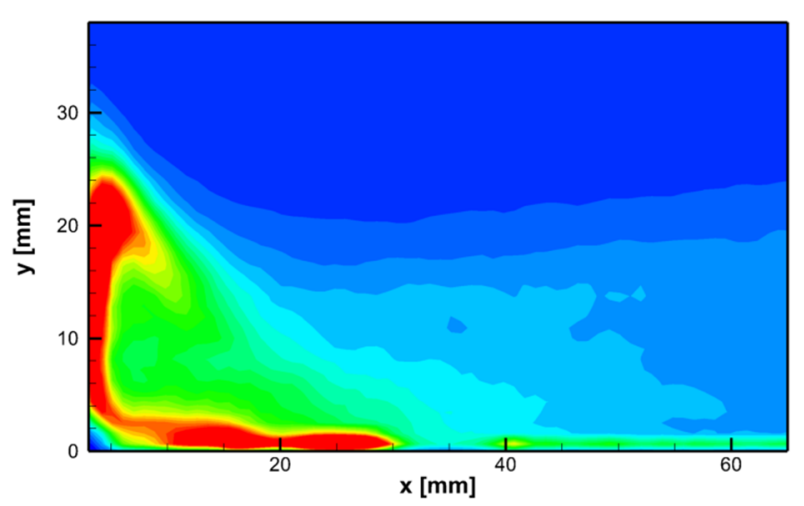

GI

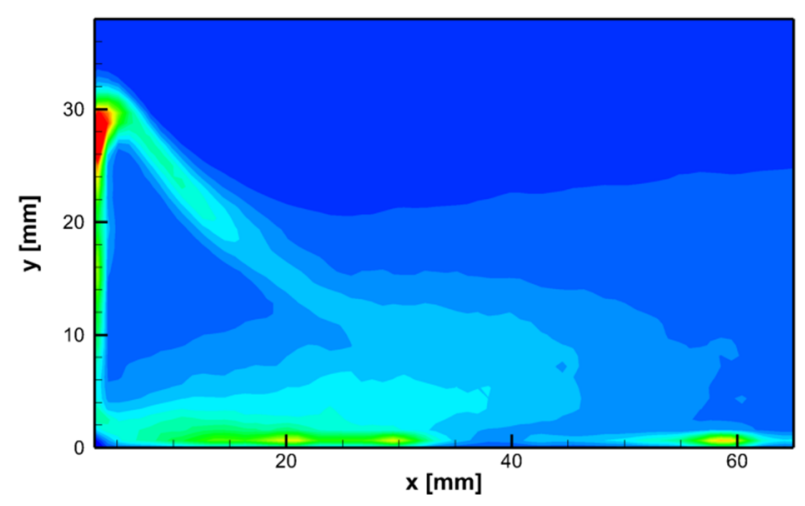

LI

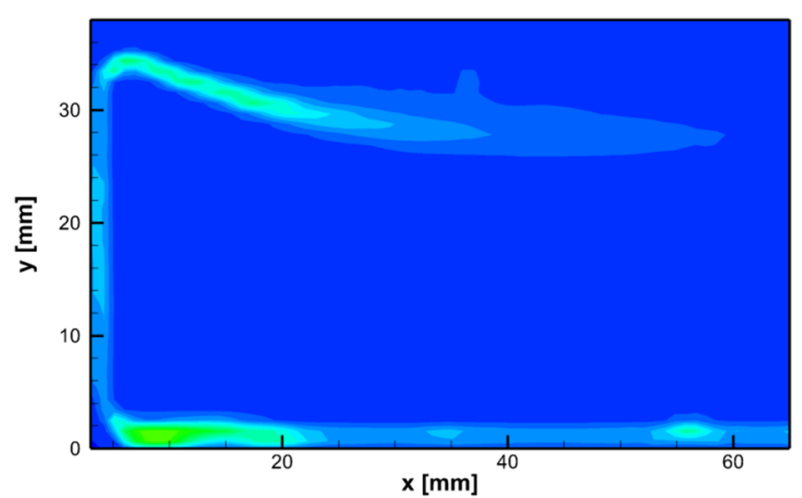

(e)

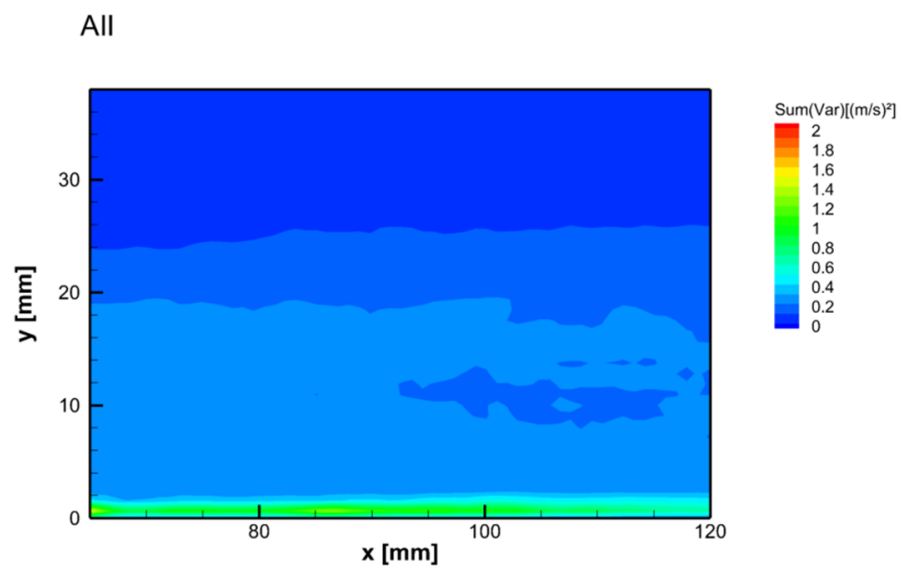

GII

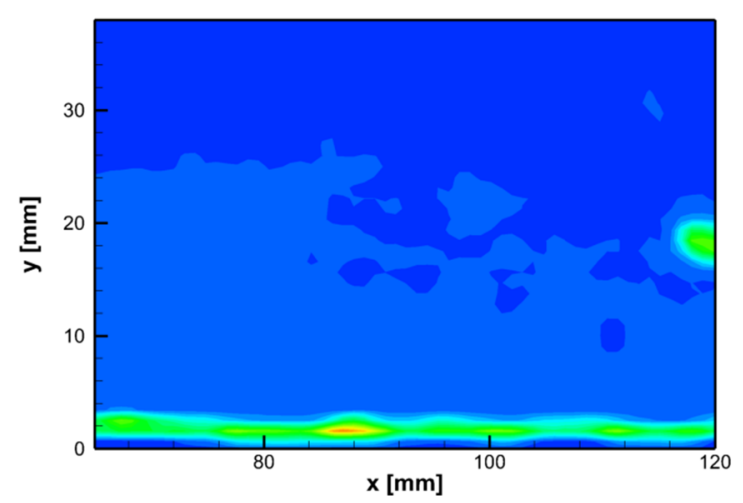

(d)

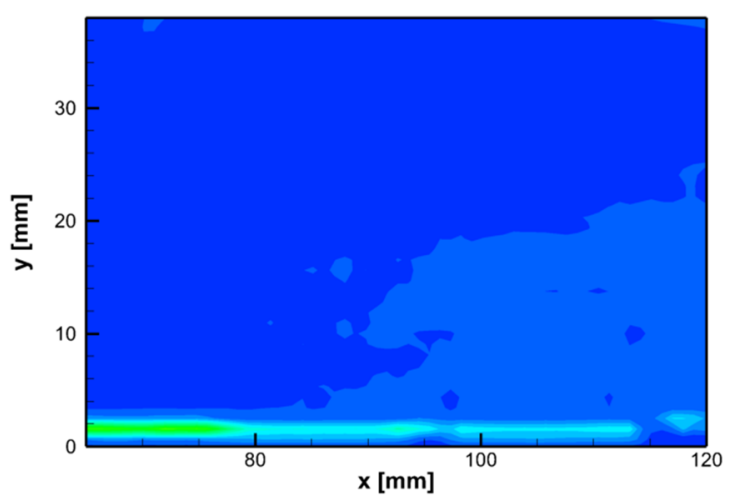

(f)

Fig. 8. Velocity components variances sum distributions in the branches A, G and L respectively: (a) AI, (b) AII, (c) GI, (d) GII, (e) LI and (f) LII.

All parameters of the selected modes includingfrequency, e-folding time and periodicity are given in Tables 1-3 respectively. The Strouhal number is determined using the mode frequency, mean velocity in the given branch calculated from a branch flow-rate (see Fig. 2) and channel width $(38 \mathrm{~mm})$. The parameters related to the flow dynamics in the branch A are shown in Table 1.
Topology of the selected modes is to be shown next. Each mode topology consists of real and imaginary parts respectively. The real part corresponds to the phase angle 0 , while the imaginary part corresponds to the phase angle $\pi / 2$, than phase $\pi$ is characterized by negative real part and the phase $3 \pi / 2$ by the negative imaginary part. The process is pseudo-periodical with decaying amplitude. 
$\mathrm{Al}$

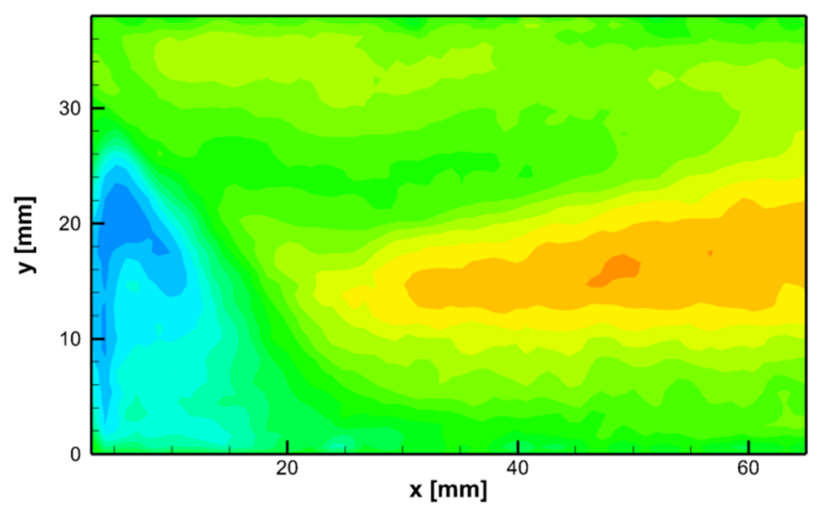

GI

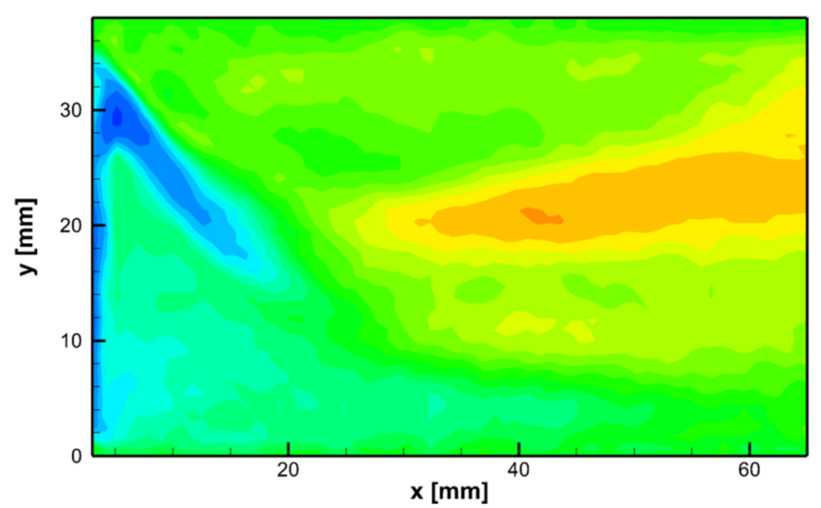

LI

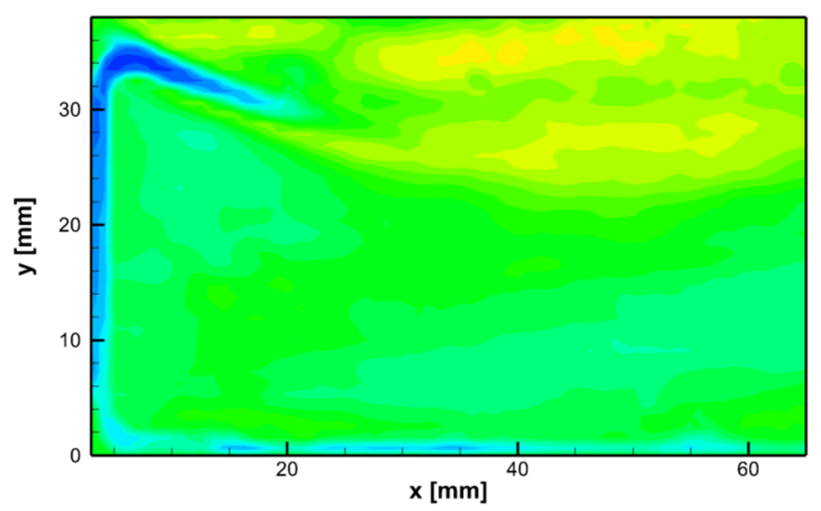

(e)
All

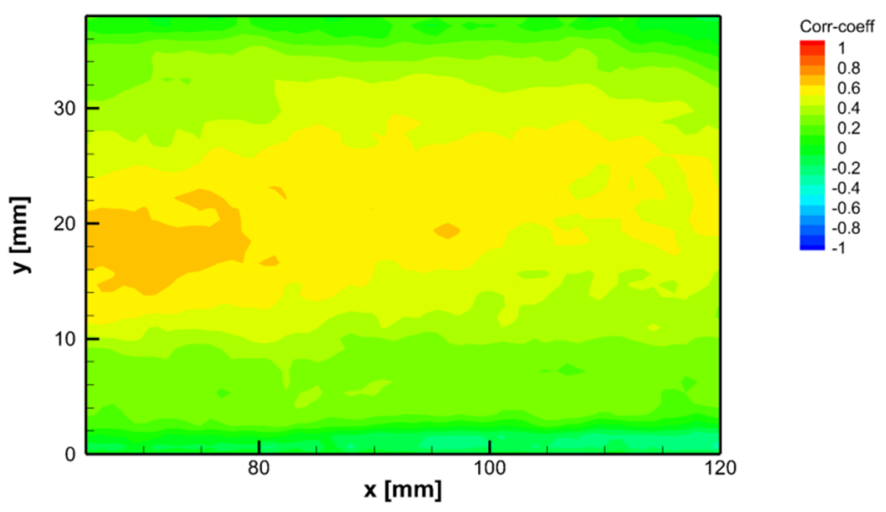

GII

(b)

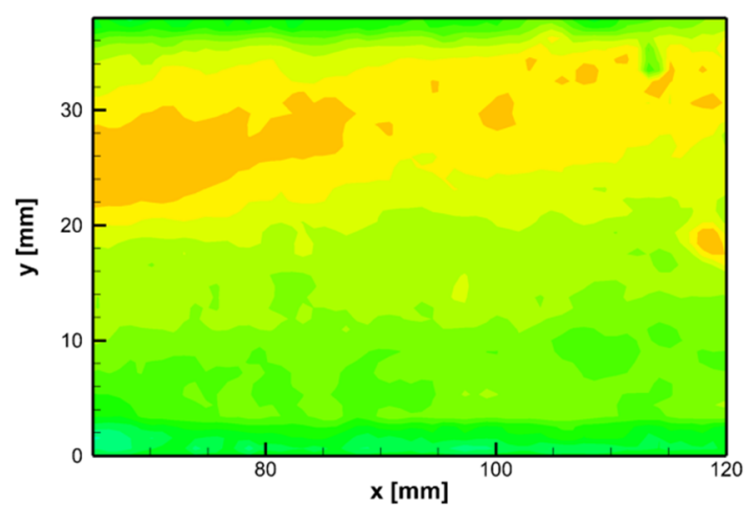

(d)

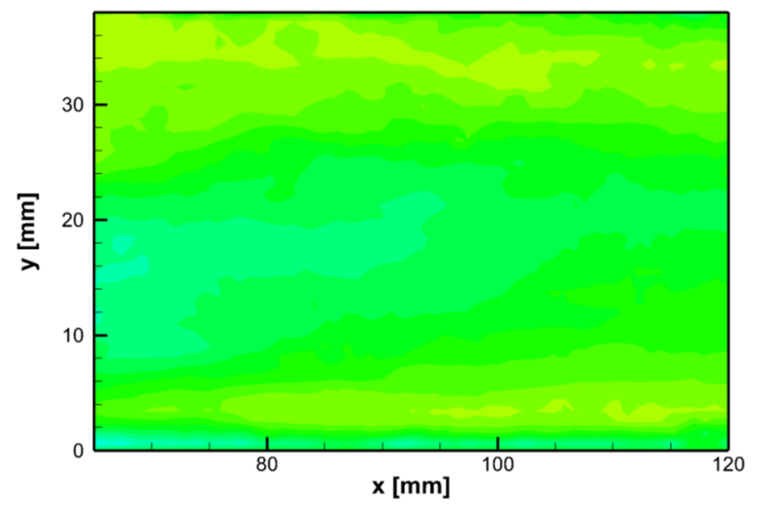

(f)

Fig. 9. Velocity components correlation coefficient distributions in the branches A, G and L, respectively: (a) AI, (b) AII, (c) GI, (d) GII, (e) LI and (f) LII.

Topology is shown as vector fields. For clearness the vector lines are added arbitrarily, for the real part in red and for the imaginary part in blue colours.

The topology of the mode 1 , branch A, zone I is shown in Figure 11.

The OPD mode 1 for AI represents the train of vortices moving in $x$ direction, spacing $s=38.4 \mathrm{~mm}$ and the period corresponding to the frequency $18.4 \mathrm{~Hz}$ is $54.34 \mathrm{~ms}$. Thus, the velocity of the vortex train is about $0.71 \mathrm{~m} / \mathrm{s}$.
Topology of the mode 2 in Figure 12 is similar to the mode 1 , however the vortices in train have smaller spacing $s=23.4 \mathrm{~mm}$ and the frequency is higher in the same time $f=42.4 \mathrm{~Hz}$. Resulting velocity is similar but higher, about $0.99 \mathrm{~m} / \mathrm{s}$. Obviously, the imaginary part of the mode topology is shifted in space about a quarter of the period.

The OPD mode 4, AI in Figure 13 is again a train of vortices propagating in the green arrow direction by the velocity $0.95 \mathrm{~m} / \mathrm{s}$. 


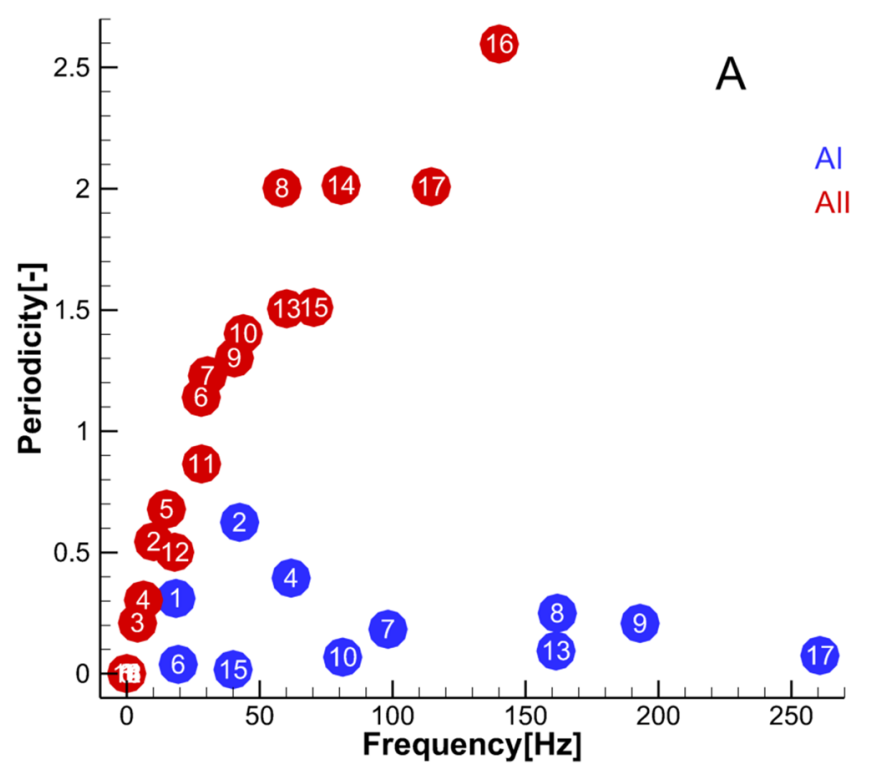

Table 1. Selected OPD modes, branch A.

Fig. 10. OPD modes, frequency and periodicity for the branch A. Zone I in blue, zone II in red.

\begin{tabular}{ccclcl}
\hline & $\begin{array}{l}\text { OPD } \\
\text { mode }\end{array}$ & $\begin{array}{l}\text { Frequency } \\
{[\mathrm{Hz}]}\end{array}$ & $\begin{array}{l}\text { Strouhal } \\
\text { No. [6] }\end{array}$ & $\begin{array}{l}\text { e-Folding } \\
{[\mathrm{ms}]}\end{array}$ & $\begin{array}{l}\text { Periodicity } \\
{[6]}\end{array}$ \\
\hline \multirow{4}{*}{ AI } & 1 & 18.4 & 0.38 & 16.82 & 0.31 \\
& 2 & 42.4 & 0.88 & 14.71 & 0.62 \\
& 4 & 61.8 & 1.28 & 6.37 & 0.39 \\
& 8 & 58.4 & 1.21 & 34.28 & 2.00 \\
AII & 14 & 80.6 & 1.66 & 24.98 & 2.01 \\
& 16 & 140.1 & 2.89 & 18.54 & 2.60 \\
& 17 & 114.5 & 2.36 & 17.53 & 2.01 \\
\hline
\end{tabular}

Al OPD 01 real

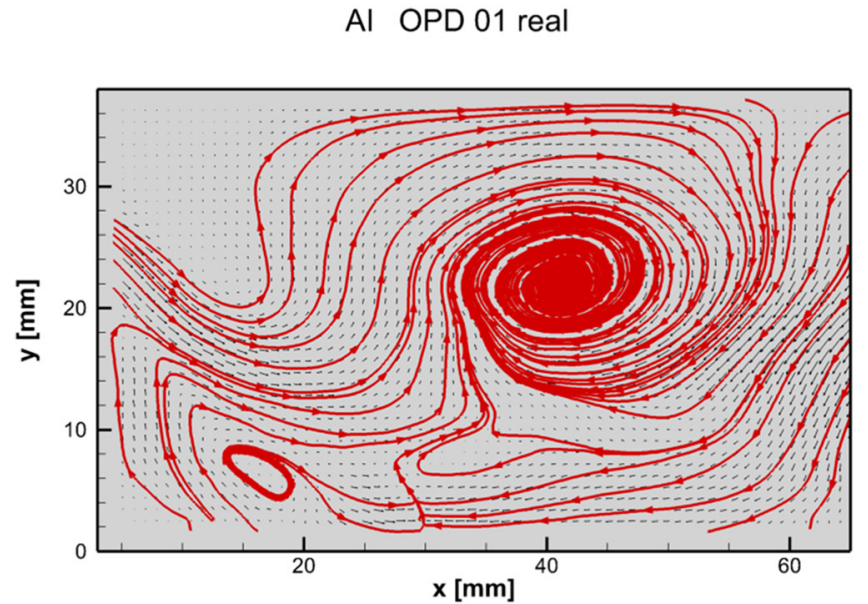

(a)
Fig. 11. Topology of the OPD mode no. 1 in the branch A, zone I, real (a) and imaginary (b) parts.

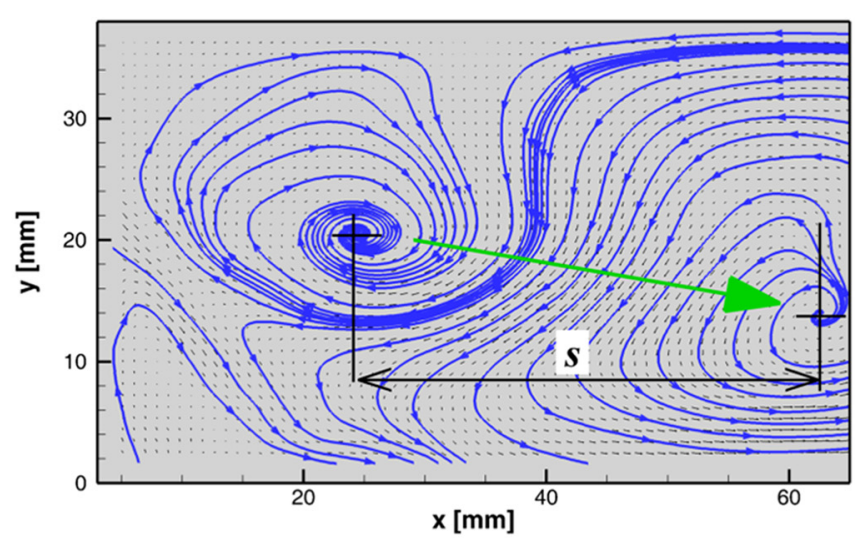

(b)
Al OPD 02 real

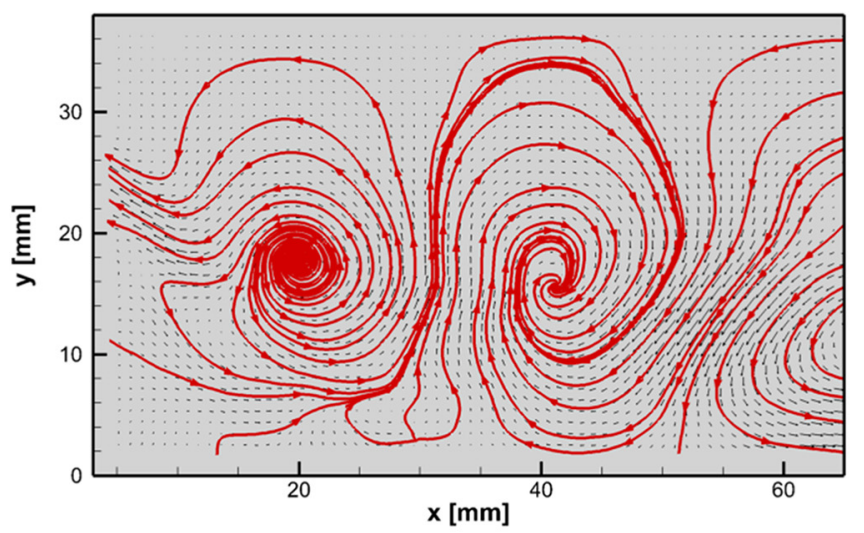

(a)
Al OPD 02 imag

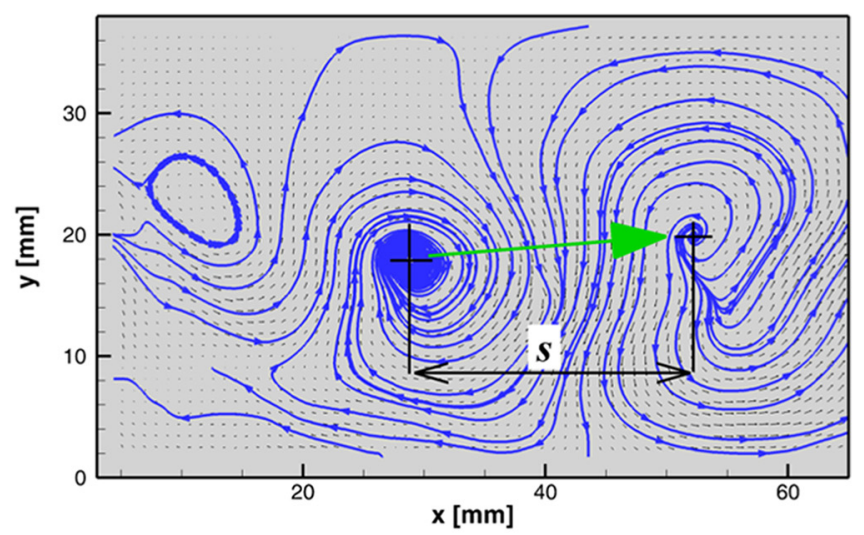

(b)

Fig. 12. Topology of the OPD mode no. 2 in the branch A, zone I, real (a) and imaginary (b) parts. 


\section{Al OPD 04 real}

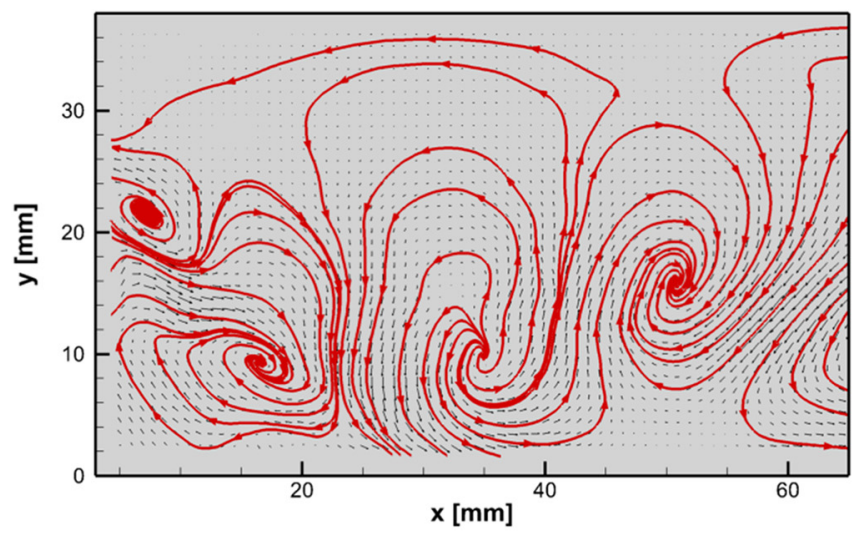

(a)
Al OPD 04 imag

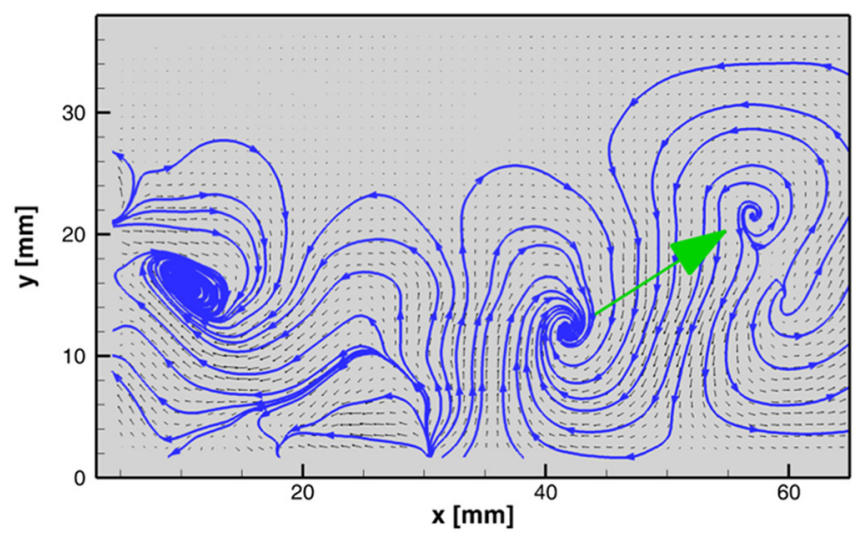

(b)

Fig. 13. Topology of the OPD mode no. 4 in the branch A, zone I, real (a) and imaginary (b) parts.

All OPD 08 real

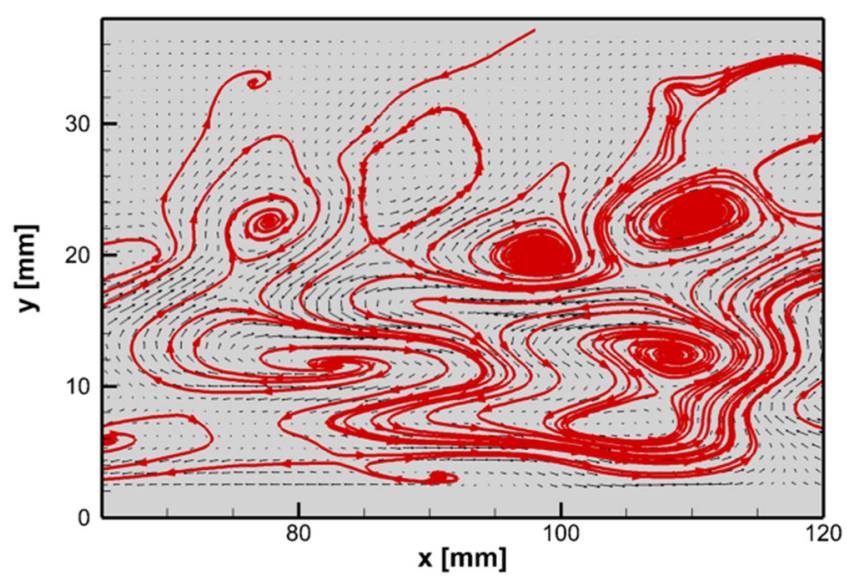

(a)

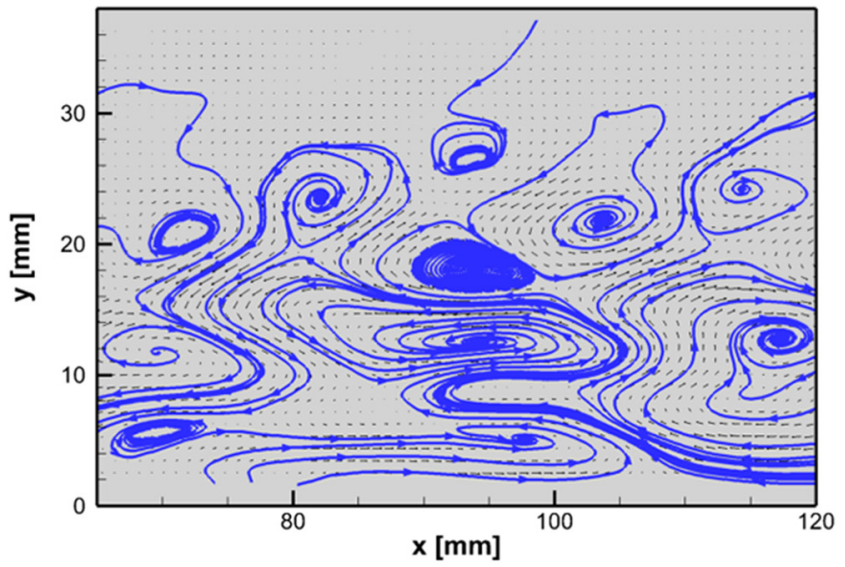

(b)

Fig. 14. Topology of the OPD mode no. 8 in the branch A, zone II, real (a) and imaginary (b) parts.

So, for the dynamics of the first zone of the branch A we could conclude, that it is populated by trains of vortices configured in a single row moving approximately in the streamwise direction $x$ with the velocity $0.7-1 \mathrm{~m} / \mathrm{s}$. The orientations of the vortices in the row are alternating.

The dynamical flow-field of the zone AII is populated by smaller vortical structures forming more complicated configuration and filling lower 2 thirds of the area. Again, the structures are propagating in waves in the streamwise direction. As an example the OPD mode AII 8 is shown in Figure 14.

The other highly periodical modes within the AII are 14, 16 and 17, in Figure 15 only real parts of the modes are shown. The modes consist of one or more rows of vortices oriented and moving in $x$ direction. The imaginary partsare very similar, only the structures are shifted in space about $1 / 4$ of the period in streamwise direction, which is the direction of movement. The typical velocities of the vortex trains within the AII are about $1.4 \mathrm{~m} / \mathrm{s}$.
Overall the flow dynamics in the first branch $\mathrm{A}$ is characterized by vortex trains propagating along the channel. In the branch inlet, zone I, the vortices are relatively big filling the whole channel, the frequency is low, Strouhal number typically smaller than 1. Further downstream, in the zone II, the instability is developed close to the back-flow region located in the lower half of the channel producing strong vortex train. Those vortices are much smaller, well developed and the frequency is much higher, Strouhal number is 1.2-2.9. Stability of pseudoperiodical vortex trains, characterized by periodicity, is much higher in the second part of the branch.

Next, the flow dynamics in the branch $G$ located in the middle of the main channel is to be shown.

In Figure 16 the frequency - periodicity graph is given. Here, in the branch G, both zones I and II exhibit highly periodical OPD modes. The relevant values for the selected modes with the periodicity higher than 1 are given in Table 2. 
All OPD 14 real

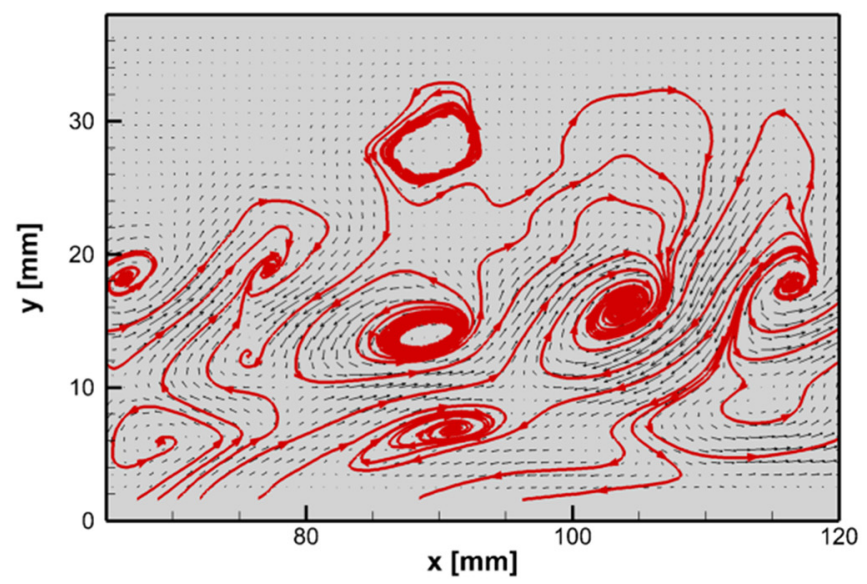

(a)

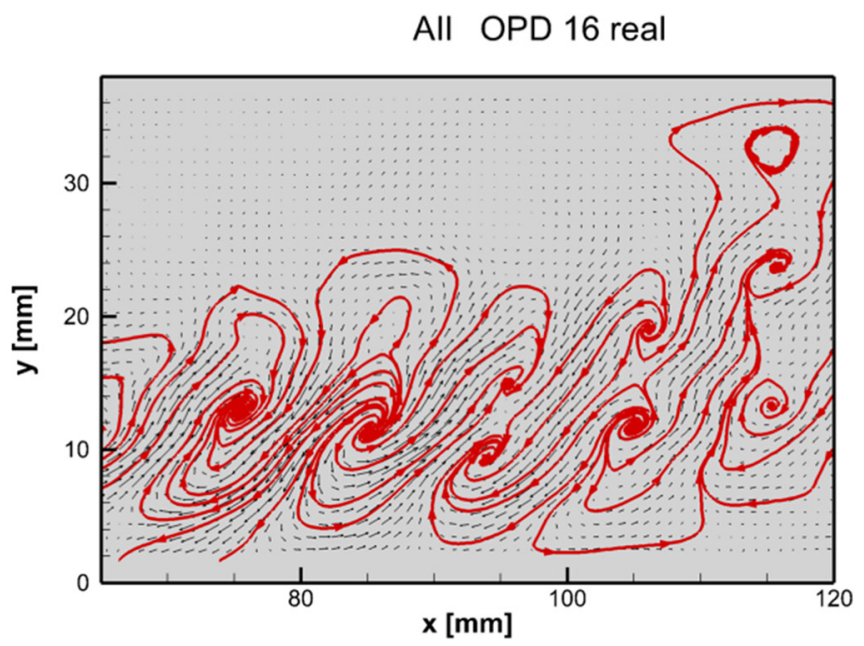

(b)

All OPD 17 real

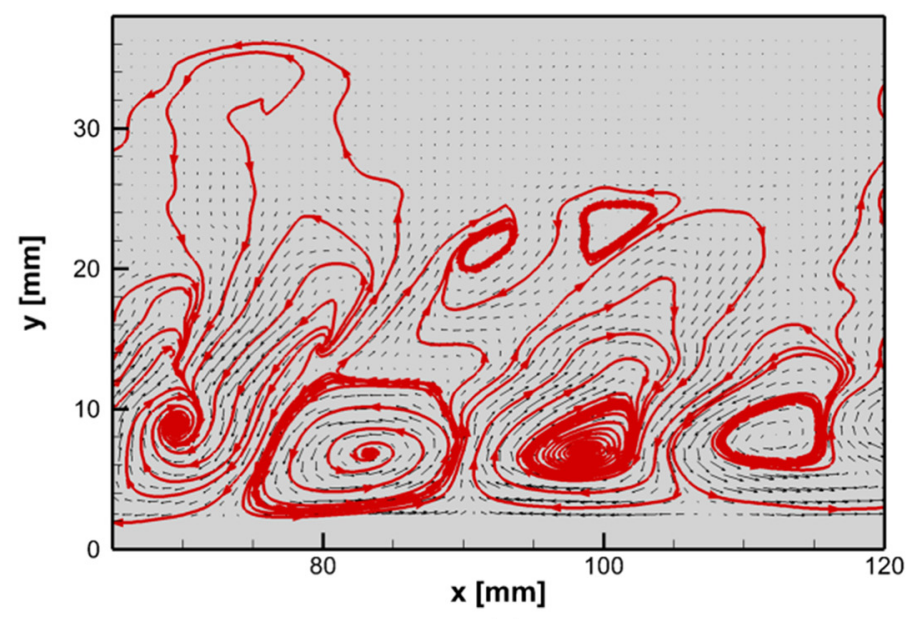

(c)

Fig. 15. Topologies of the selected OPD modes in the branch A, zone II, real parts (a) 14, (b) 16 and (c) 17 .

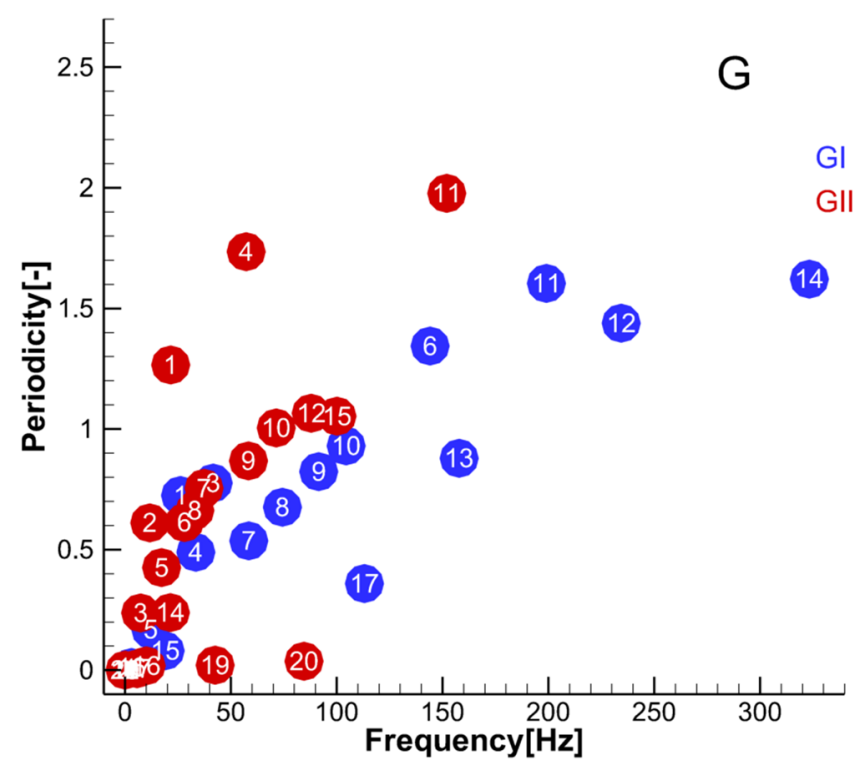

Fig. 16. OPD modes, frequency and periodicity for the branch G. Zone I in blue, zone II in red. 
Table 2. Selected OPD modes, branch G.

\begin{tabular}{ccllcc}
\hline & OPD mode & Frequency $[\mathrm{Hz}]$ & Strouhal No. [6] & e-Folding [ms] & Periodicity [6] \\
\hline \multirow{4}{*}{ GI } & 6 & 144.2 & 2.59 & 9.32 & 1.34 \\
& 11 & 199.1 & 3.58 & 8.05 & 1.60 \\
& 12 & 234.5 & 4.22 & 6.13 & 1.44 \\
& 14 & 323.2 & 5.81 & 5.02 & 1.62 \\
GII & 1 & 21.5 & 0.39 & 58.71 & 1.27 \\
& 4 & 57.2 & 1.03 & 30.31 & 1.73 \\
& 11 & 152.0 & 2.73 & 13.00 & 1.98 \\
\hline
\end{tabular}

GI OPD 06 real

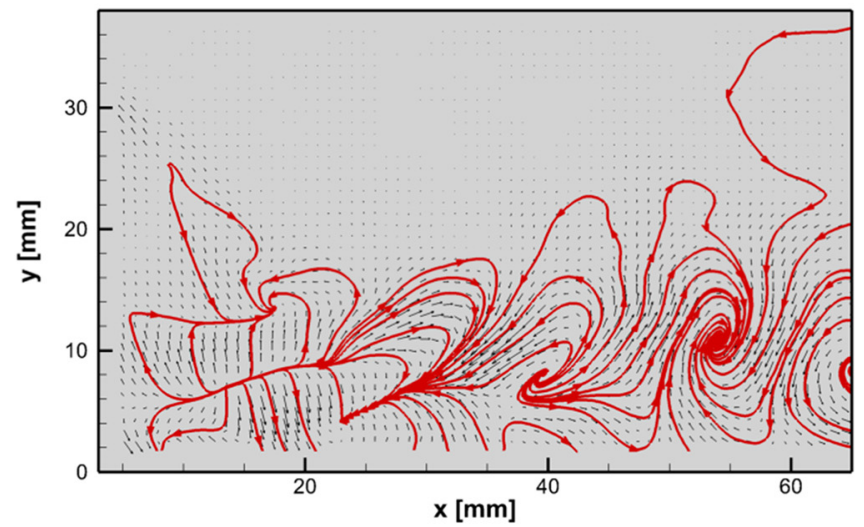

(a)

GI OPD 12 real

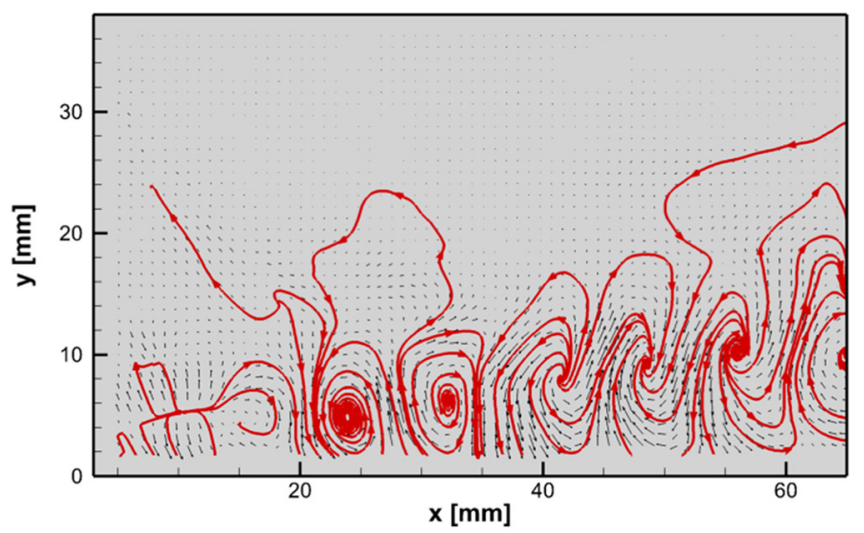

(c)

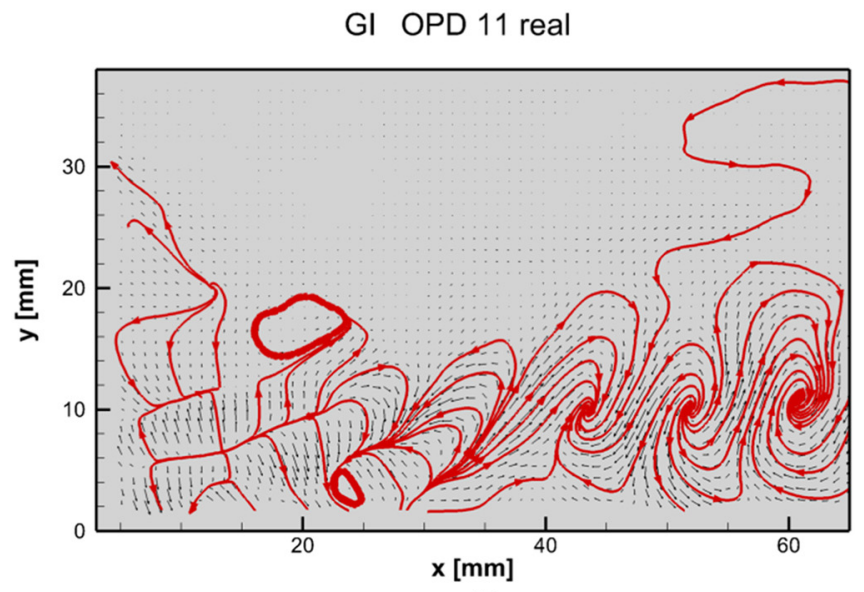

(b)

GI OPD 14 real

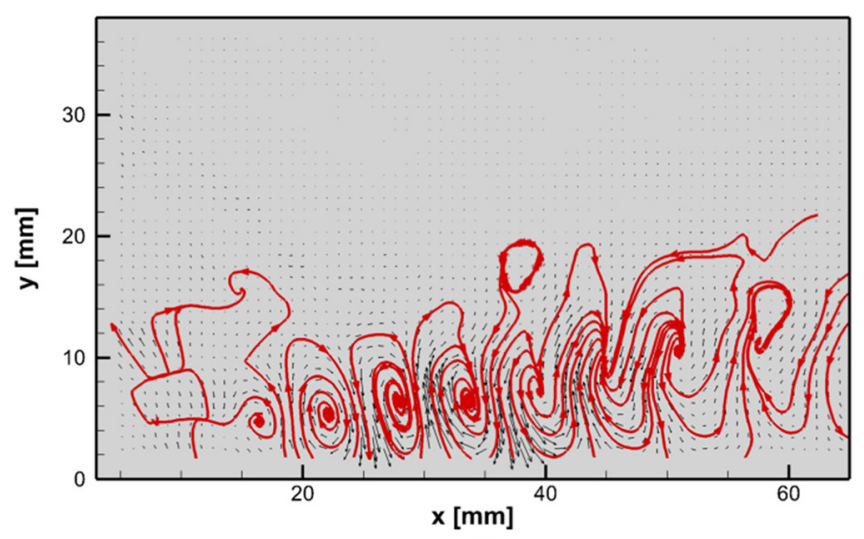

(d)

Fig. 17. Topologies of the selected OPD modes in the branch G, zone I, real parts, (a) 6, (b) 11 , (c) 12 and (d) 14.

The topology of the selected modes is introduced bellow. In the zone I of the branch $\mathrm{G}$ the typical topology is represent consisting of vortex train with alternating orientation located in the lower domain third. Only the real parts of the modes are to be shown here, as the imaginary parts are only shifted in space, as mentioned above.

In Figure 17 there are modes 6, 11, 12 and 14, the typical convection velocity of vortices is about $1.5 \mathrm{~m} / \mathrm{s}$. The frequencies relatively high, the Strouhal number between 2.6 and 5.8.
The zone II of the branch $\mathrm{G}$ is to be presented now. The mode 1 is characterized by a train of big vortices filling the whole domain. Train of the smaller vortices in $x$ direction is detected in the mode no. 11, see Figure 18. The velocity of the vortices is $0.75 \mathrm{~m} / \mathrm{s}$ for the mode no. 1 and $1.7 \mathrm{~m} / \mathrm{s}$ for the mode 11 .

The mode 4 , branchG, zone II is characterized by two rows of vortices, the first in the upper half consisting of 3 vortices and the second in the lower half consisting of 2 vortices only. Both systems are moving in the $x$ direction, however the propagation velocity is different: $0.9 \mathrm{~m} / \mathrm{s}$ for 
GII OPD 01 real

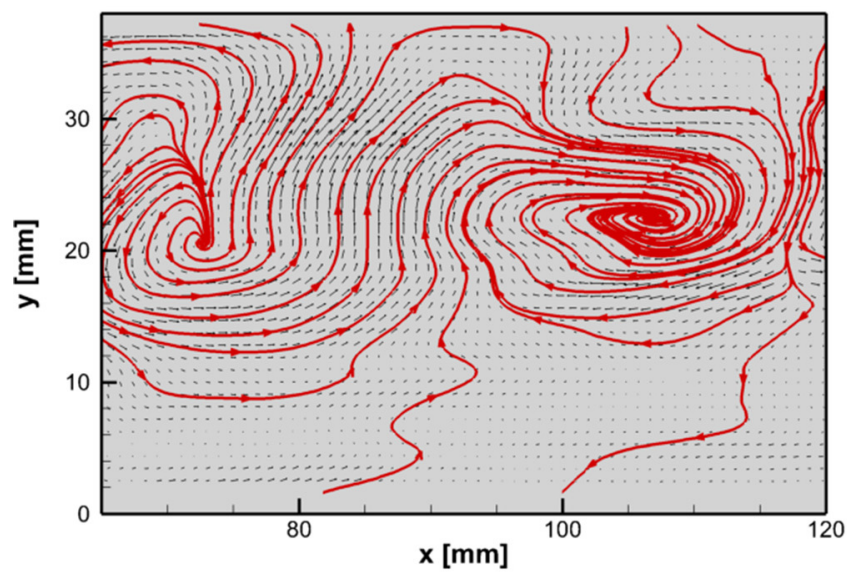

(a)

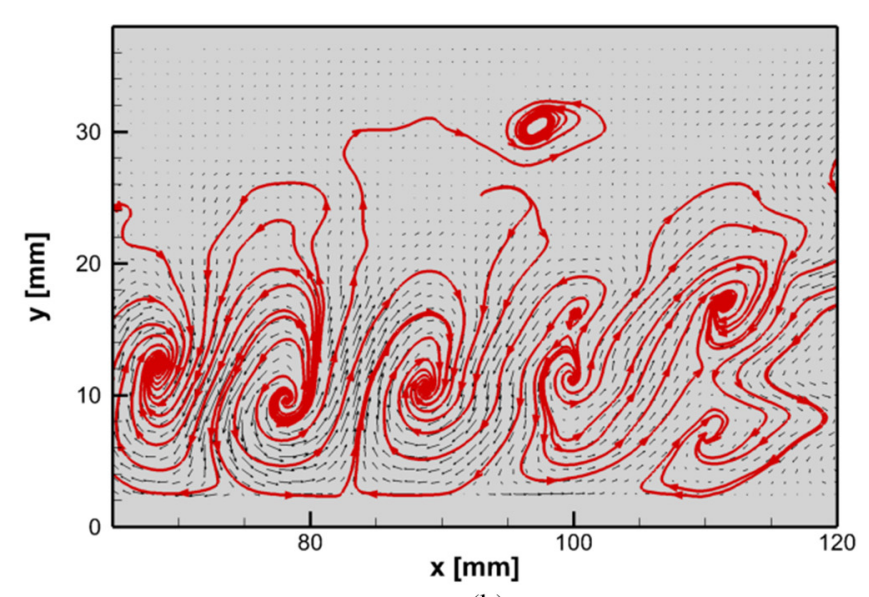

(b)

Fig. 18. Topologies of the selected OPD modes in the branch G, zone II, real parts, numbers (a) 1 and (b) 11 .

GII OPD 04 real

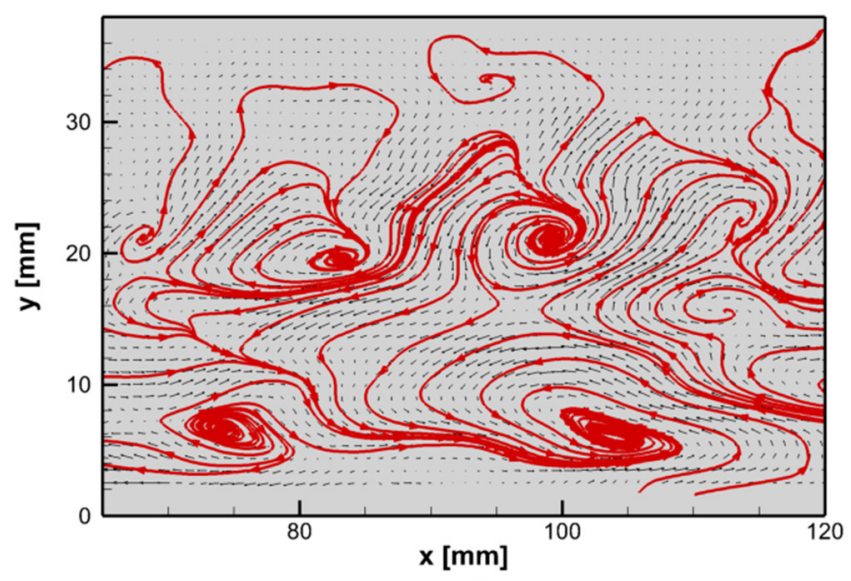

(a)

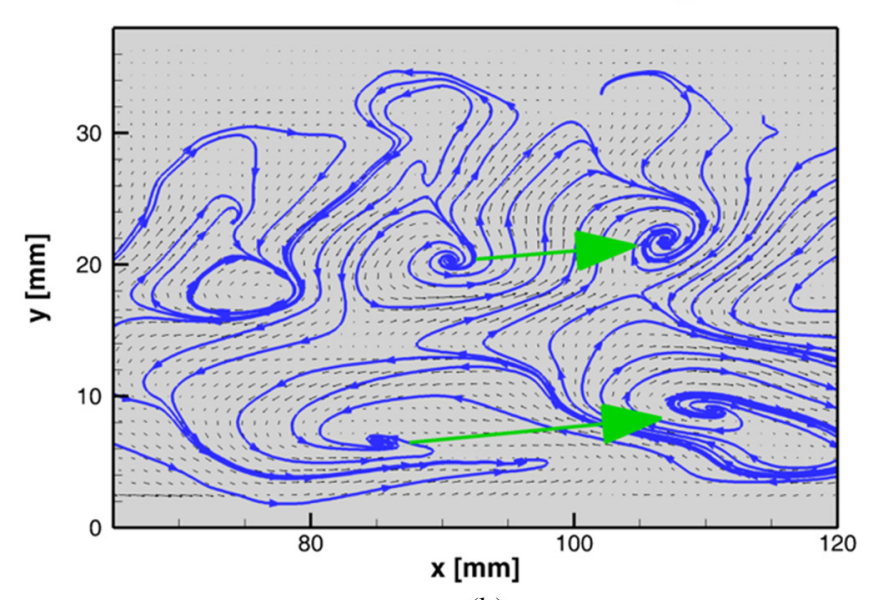

(b)

Fig. 19. Topology of the OPD mode no. 4 in the branch G, zone II, real (a) and imaginary (b) parts.

the upper system, while $1.7 \mathrm{~m} / \mathrm{s}$ for the lower system. Both real and imaginary part of the mode 4 , branchG, zone II topology are shown in Figure 19.

The dynamical situation in the branch $\mathrm{G}$ is in some aspect opposite to this in the branch $\mathrm{A}$. In the branch $\mathrm{G}$, in the inlet the frequencies of vortex trains are very high, the Strouhal number reaches 5.8. The structures are located in the lower third of the channel close to the wall. Further downstream, in the zone II, the vortex trains form two-row structure filling the whole channel (the mode 4). The frequencies are lower.

Last branch to be analysed for the flow dynamics is the branch L, the last but one in the order. In the branch $\mathrm{L}$ the flowdynamics is weak, the flow is rather steady. The frequency-periodicity graph is in Figure 20 and the selected modes are specified in Table 3.

The modes in the branch L, zone I, nos. 3 and 10 are formed by system of saddle lines of oblique orientation see the real parts of the modes topology in Figure 21a and b. In the upper part there is an indication of vortices. The saddle lines are moving in the vertical direction, perpen-

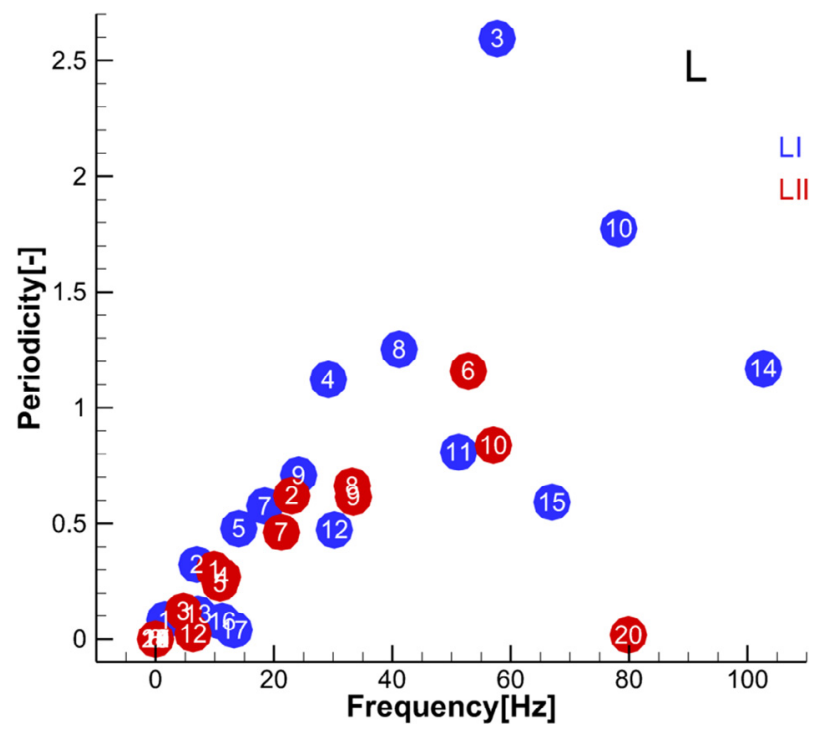

Fig. 20. OPD modes, frequency and periodicity for the branch L. Zone I in blue, zone II in red. 
Table 3. Selected OPD modes, branch L.

\begin{tabular}{lcclll}
\hline & OPD mode & Frequency $[\mathrm{Hz}]$ & Strouhal No. [6] & e-Folding $[\mathrm{ms}]$ & Periodicity [6] \\
\hline \multirow{3}{*}{ LI } & 3 & 57.7 & 0.72 & 44.95 & 2.59 \\
& 10 & 78.3 & 0.98 & 29.31 & 1.77 \\
\multirow{2}{*}{ LII } & 14 & 102.7 & 1.28 & 11.38 & 1.17 \\
& 6 & 52.8 & 0.66 & 21.93 & 0.84 \\
\hline
\end{tabular}

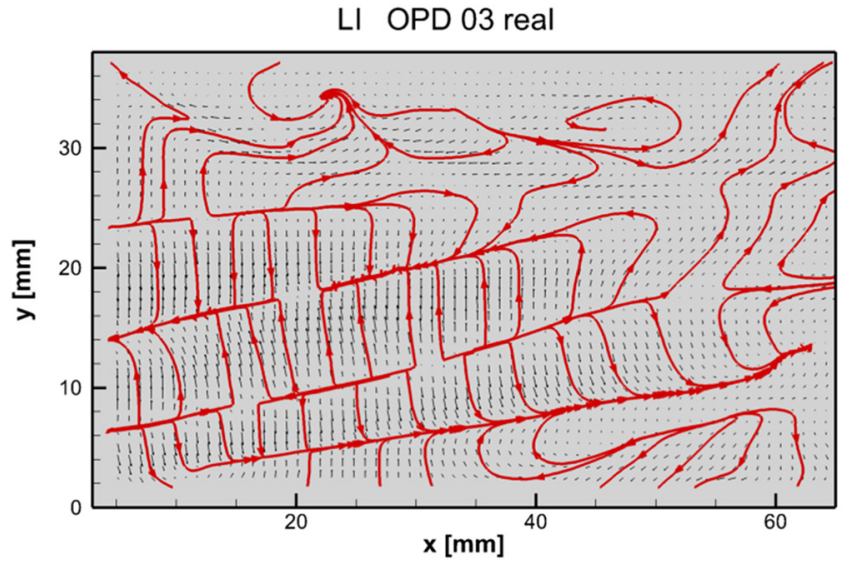

(a)

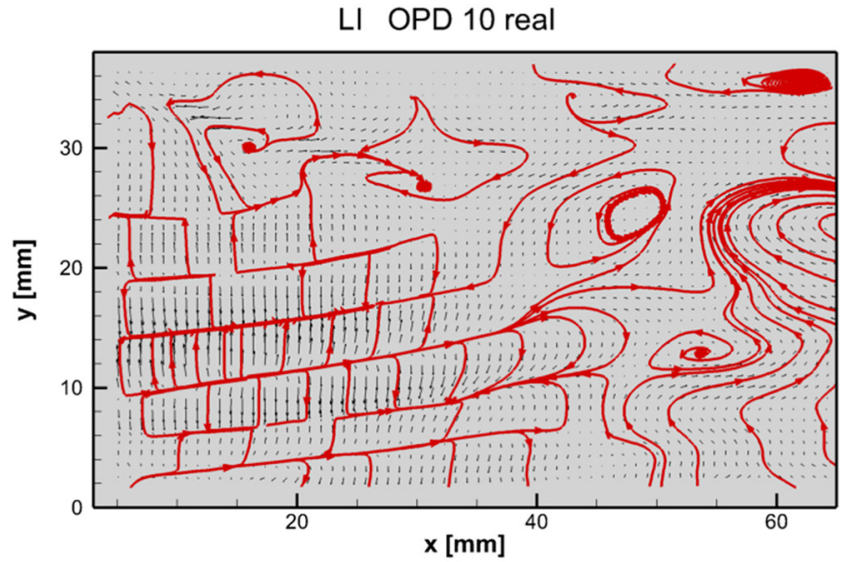

(b)

LI OPD 14 real

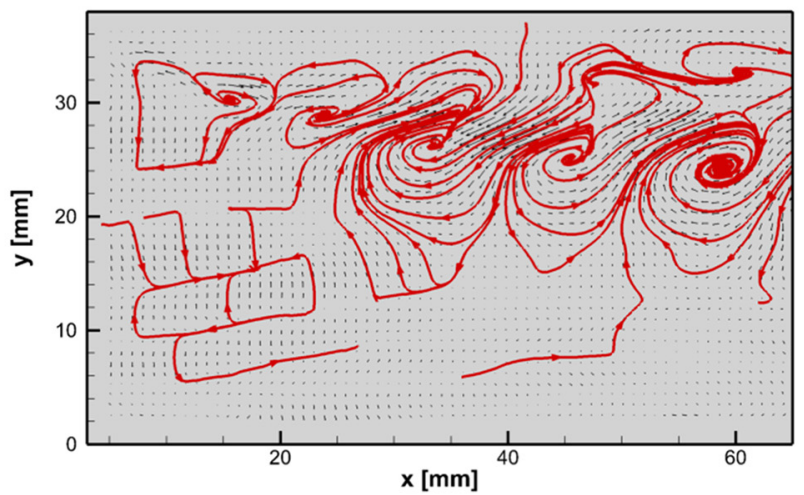

(c)

Fig. 21. Topologies of the selected OPD modes in the L branch, zone I, real parts, (a) 3, (b) 10, (c) 14 .

dicularly to the flow. The mode 3 is very stable and well pronounced, the frequency is moderate, the Strouhal number 0.72 .

The vortex train is well detectable in the branch $\mathrm{L}$, zone I mode no. 14, it is located in the upper half of the domain, velocity of propagation in streamwise direction about $1.36 \mathrm{~m} / \mathrm{s}$.

The mode no. 6 in the branch L, zone II represents pairs of contra-rotating vortices travelling in the middle of the domain in the $x$ direction, the velocity is close to $1 \mathrm{~m} / \mathrm{s}$, see Figure $22 \mathrm{a}$ and $\mathrm{b}$.

The mode no. 10 in the branch L, zone II is shown in Figure 23a and b, both real and imaginary parts. The mode consists of elongated vortices stretched in oblique direction. The vortices are convected perpendicularly to the elongation direction - see the green arrow in
Figure 23b. The convection velocity is very small, about $0.28 \mathrm{~m} / \mathrm{s}$.

In the $\mathrm{L}$ branch, the last but one, typical dynamic of moving structures in transversal direction has been detected. The typical dynamical structures are in the form of elongated vortices and the predominant saddle lines. The corresponding frequencies are moderate, typical Strouhal number below 1 .

The heat transfer process between the wall and fluid is strongly affected by the flow structure close to the wall. Both mean velocity and the flow dynamics play important role in this process, as shown e.g. in [10].

In general, the stable, well profound vortical trains occurring close to the walls and high mean velocity will promote heat transfer. In our complex case such regions could be detected close to the lower walls of the AII region 


\section{OPD 06 real}

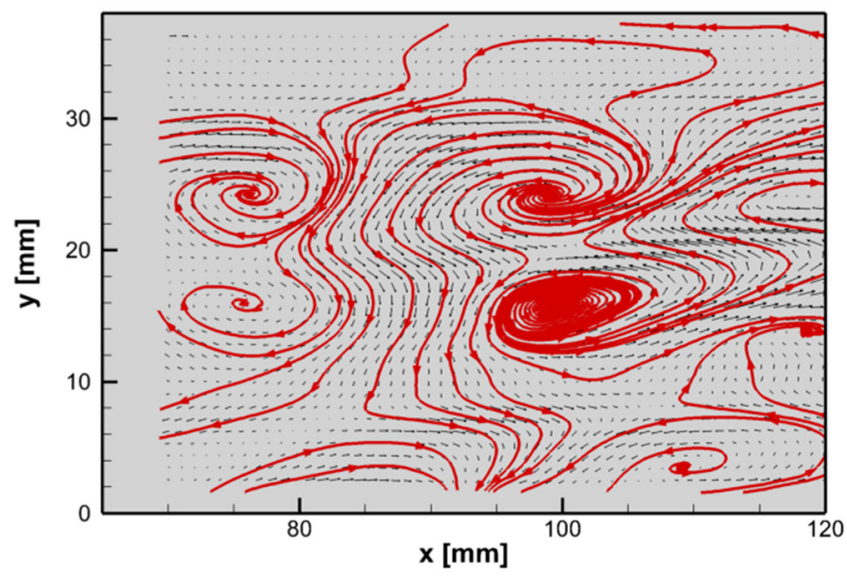

(a)

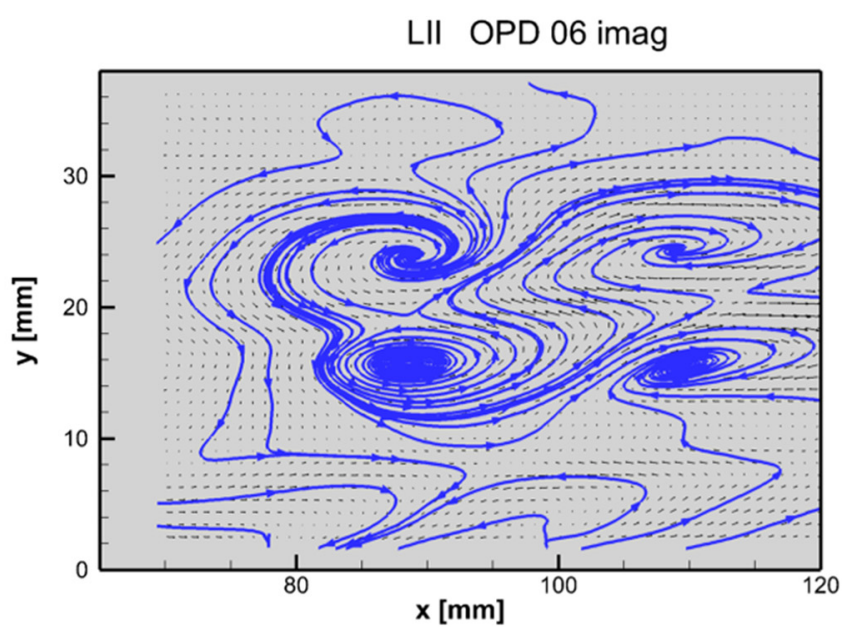

(b)

Fig. 22. Topology of the OPD mode in the branch L, zone II, number 6, real (a) and imaginary (b) parts.

LII OPD 10 real

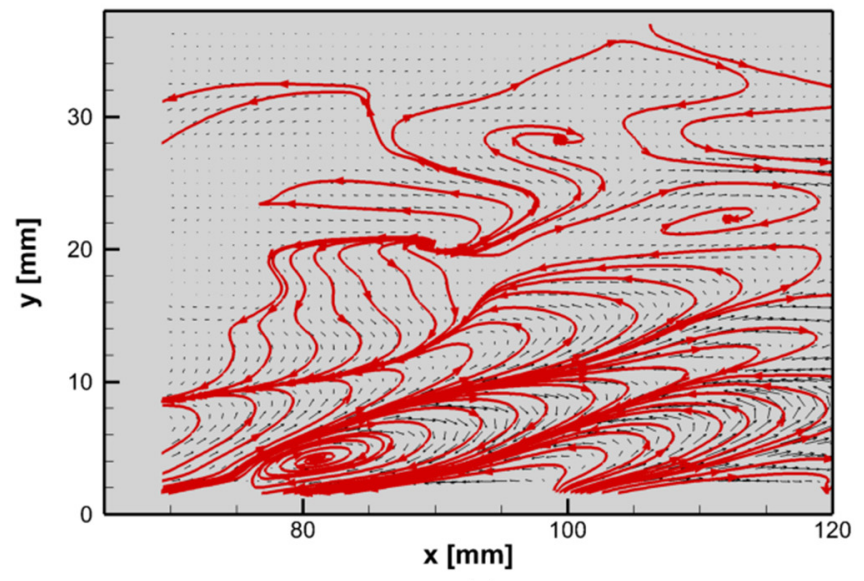

(a)

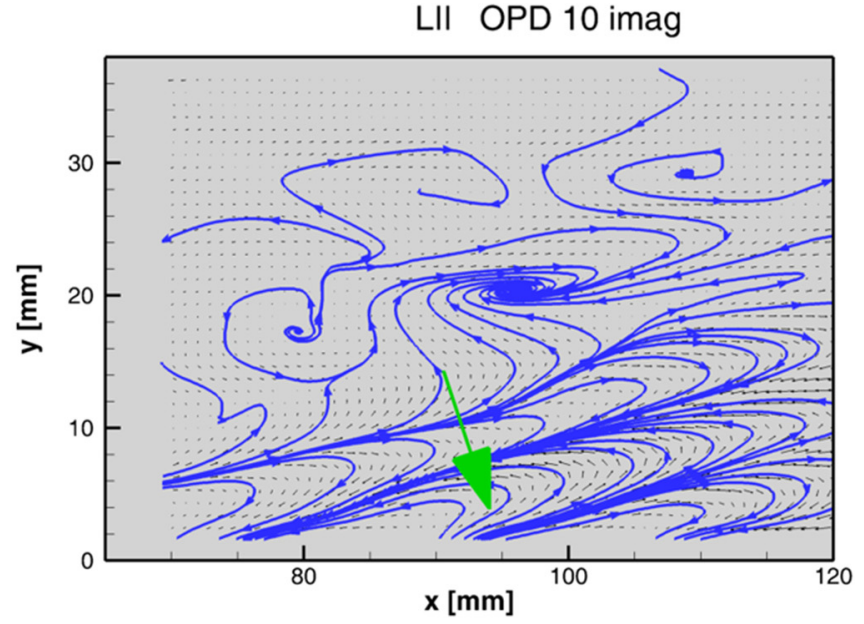

(b)

Fig. 23. Topology of the OPD mode in the branch L, zone II, number 10, real (a) and imaginary (b) parts.

and all the $\mathrm{G}$ branch. In the case of the $\mathrm{L}$ branch, both lower and upper walls are surrounded by dynamical structures and thus in good thermal communication with the flow.

On the other hand, the low-velocity flow-regions with weak fluctuations prevent the heat transfer. The typical such regions are the separation regions affecting the upper walls of the A and $\mathrm{G}$ branch channels.

\section{Conclusions}

In the paper the flow structure of selected branches of a sample branched channel is shown as a result of PIV experiments. The flow is separated creating a back-flow region. In the shear layer created, the instability effects take place.

Both statistical methods based on averaging and special methods for vector field frequency analysis are applied. Typical pseudo-periodic dynamical structures are detected. The typical dynamical structures are trains of vortices of alternative orientation aligned in the $x$ direction and propagating in the streamwise direction and moving saddle lines. However, the dynamical structures differ substantially in individual branches.

The flow dynamics in the branches depends strongly on the position on the main channel. Three distinct regions were identified in this context.

Close to the main channel inlet, the branches flowdynamics is characterized by vortex trains propagating along the channel. In the branch inlet the vortices are relatively big filling the whole channel, the frequency is low, Strouhal number typically smaller than 1. Further downstream the instability is developed close to the back-flow region located in the lower half of the channel producing strong vortex train. Those vortices are much smaller, well developed and the frequency is much higher than in the first partof the branch. Stability of pseudoperiodical vortex trains, characterized by periodicity value, is much higher in the second part of the branch.

In the region of the main channel middle part, the dynamical situation in branches is nearly opposite to this in the inlet branches. Here, close to the branch inlet, the frequencies of vortex trains are very high, Strouhal 
numbers up to 5.8. The structures are located in the lower third of the channel close to the lower wall. Further downstream the vortex trains form two-row structure filling the whole channel and the frequencies are again lower.

Approaching the main channel end, the typical flow dynamics in the branches is characterized by the structures moving in transversal direction. The typical dynamical structures are in the form of the predominant saddle lines and elongated vortices. The corresponding frequencies are moderate, Strouhal numbers bellow 1.

The flow-field close to the channel walls affects heat transfer process between the wall and fluid, high velocities and intensive fluctuations generated by dynamical structures promote the heat transfer.

\section{Nomenclature}

$\begin{array}{ll}f[\mathrm{~Hz}] & \text { Frequency } \\ p[1] & \text { Periodicity } \\ s[\mathrm{~m}] & \text { Spacing } \\ x, y[\mathrm{~m}] & \text { Cartesian coordinates } \\ x_{\mathrm{ra}}[\mathrm{m}] & \text { Reattachment position } \\ x_{\mathrm{c}}, y_{\mathrm{c}}[\mathrm{m}] & \text { Position of vortex centre } \\ \mathrm{A}, \mathrm{B}, \mathrm{C}, \mathrm{D}, \mathrm{E}, \mathrm{F}, \mathrm{G}, & \text { Branches indicators (11) } \\ \mathrm{H}, \mathrm{I}, \mathrm{J}, \mathrm{K}, \mathrm{L}, \mathrm{M} & \\ \mathrm{I}, \mathrm{II} & \text { Zones }(2) \\ \text { OPD } & \text { Oscillation Pattern Decomposition } \\ \text { PIV } & \text { Particle Image Velocimetry }\end{array}$

This work was supported by the Grant Agency of the Czech Republic, projects Nos. 17-01088S and 19-02288J and by the Technology Agency of the Czech Republic, project No. TK03020057.

\section{References}

[1] V. Uruba, P. Procházka, V. Skála, Flow in branched channel, Topical Problems of Fluid Mechanics 2018, 299-306 (2018)

[2] I.E. Idelchik, Handbook of Hydraulic Resistance, Jaico Publishing House, Mumbai, 2005

[3] J. Nikuradse, Untersuchungen über die Geschwindigkeitsverteilung in turbulenten Strijmungen, $\mathrm{PhD}$ thesis, Götingen, 1926

[4] V. Uruba, O. Hladík, P. Jonáš, Dynamics of secondary vortices in turbulent channel flow, Journal of Physics: Conference Series 318, 062021 (2011)

[5] A. Bottaro, H. Soueid, B. Galletti, Formation of secondary vortices in turbulent square-duct flow, AIAA Journal 44, 803-811 (2006)

[6] L. Beneš, P. Louda, K. Kozel, R. Keslerová, J. Štigler, Numerical simulations of flow through channels with T-junction, Applied Mathematics and Computation 219, 7225-7235 (2013)

[7] P. Louda, K. Kozel, J. Příhoda, L. Beněs, T. Kopáček, Numerical solution of incompressible flow through branched channels, Computers \& Fluids, 46, 318-324 (2011)

[8] V. Uruba, P. Procházka, V. Skála, Dynamics of flow in a branch of a branching channel, MATEC Web of Conferences, 168, 2018, Article number 05001, 21st International Scientific Conference - The Application of Experimental and Numerical Methods in Fluid Mechanics and Energy, AEaNMiFMaE 2018; Wellness Hotel Diplomat Rajecke Teplice; Slovakia; 25 April 2018 through 27 April 2018; Code 136770 (2018)

[9] V. Uruba, Near wake dynamics around a vibrating airfoil by means of PIV and oscillation pattern decomposition at reynolds number of 65000 , Journal of Fluids and Structures 55, 372-383 (2015)

[10] B. Sundén, Introduction to Heat Transfer, WIT Press, 2012

Cite this article as: V. Uruba, P. Procházka, V. Skála, Dynamics of flow in a branching channel, Mechanics \& Industry 22, 25 $(2021)$ 